\title{
Developmental depression-facilitation shift controls excitation-inhibition balance
}

3 David W. Jia ${ }^{1}$, Rui Ponte Costa ${ }^{1,2^{*}}$, Tim P. Vogels ${ }^{1,3^{*}}$

$4 \quad{ }^{1}$ Centre for Neural Circuits and Behaviour, Department of Physiology, Anatomy and Genetics, University

5 of Oxford, United Kingdom; ${ }^{2}$ Bristol Computational Neuroscience Unit, SCEEM, Faculty of Engineering,

- University of Bristol, United Kingdom; ${ }^{3}$ Institute of Science and Technology, Klosterneuburg, Austria

${ }^{*}$ Co-senior authors.

For correspondence: rui.costa@bristol.ac.uk (RPC) 
Using computational models, we show how short-term plasticity can complement and even control the expression of inhibitory long-term plasticity, thus acting as a gating mechanism for the emergence of excitation-inhibition balance across development. In particular, we show that short-term depression is critical to maintain stable neural activity even with flat inhibitory tuning curves in young animals ${ }^{25}$. Further, the gradual shift to short-term facilitation, as observed throughout development ${ }^{8-10,13-15}$ allows for excitatory-inhibitory balance to emerge. We show that this developmental control of STP shapes the properties of neuronal dynamics, making neural responses more diverse and postsynaptic spike timings more precise over the course of maturation. Finally, the maturation of STP in our model leads to synapse-based working memory properties in a El balanced neuron model.

\section{Results}

Changes in short-term plasticity (STP) are a hallmark of neural development ${ }^{8,12,31}$, but their impact on neuronal dynamics has remained unclear. Here, we study the effects of short-term plasticity in congruence with long-term inhibitory plasticity in a developing neuron model, and show that STP can play a crucial role in young neurons, compensating for a lack of inhibitory tuning. Moreover, gradual change of excitatory STP from depression to facilitation over development allows for excitatory-inhibitory balance to develop in the neuron while guaranteeing stable response properties.

To investigate these effects, we built a model of a simple feedforward network with a single conductance-based integrate-and-fire neuron receiving inputs from 800 excitatory and 200 inhibitory afferents ${ }^{21}$. To emulate heterogeneous inputs we modelled eight different pathways (Fig. 1a) each with 100 excitatory and 25 inhibitory synapses, whose activity is determined by a time-varying rate signal (Methods). Excitatory and inhibitory synapses were modu-

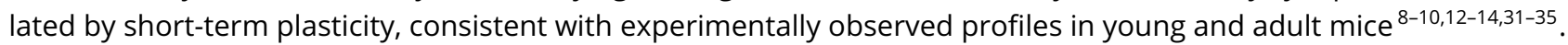
Inhibitory synapses additionally experienced long-term plasticity (ISP) ${ }^{19,22}$. Excitatory afferents were tuned according to experimentally observed receptive fields, while inhibitory baseline weights were initially flat (Fig. 1b, see also ${ }^{25}$ )

Inhibitory long-term synaptic plasticity working on a time-scale of hours has been suggested to underlie excitationinhibition (E-I) balance in cortical networks ${ }^{19,21,22}$. The slow nature of long-term synaptic plasticity is consistent with the gradual and slow development of E-I balance over multiple days from young to adult animals ${ }^{25}$ (Fig. 1b). However, the lack of detailed balance in young animals could lead to unstable, unnaturally high activity (Fig. 1c,e). Increased learning rates, on the other hand, lead to unstable learning ${ }^{36,37}$.

Short-term plasticity can offer an elegant solution to maintain low firing rates throughout development. To this end, we added experimentally observed ${ }^{4,6,7}$ short-term depression to all afferent synapses using a standard TsodyksMarkram model ${ }^{16}$ (Methods). In contrast with the ISP-alone model, the addition of an appropriate STP profile that features short-term depression at the excitatory synapses, led to lower firing rates in the 'young' model, despite unbalanced excitation-inhibition (Fig. 1d,e).

Notably, the low postsynaptic firing rates that resulted from short-term depression in the excitatory afferents effectively prevented long-term plasticity from tuning inhibitory tuning curves as has been observed in adult animals (Fig. $1 \mathrm{~b} ;{ }^{25}$ ). As we will see below, the shift of short-term plasticity profiles over the course of development ${ }^{8,12,31}$ allowed the gradual tuning of inhibition in ageing animals.

\section{Gradual depression-to-facilitation shift enables stable activity over development}

Next we studied how the developmental changes of short-term depression (STD) to short-term facilitation (STF) in excitatory synapses ${ }^{8-10,12-14,31-35}$ may aid the tuning of inhibitory synapses by way of long-term plasticity, and provide stable postsynaptic firing rates throughout the process.

To simulate ageing in our model, we devised an algorithm that slowly changed the STP parameters between young and adult profiles fitted to experimental data (Fig. 2a; Methods). The algorithm monitored average postsynaptic firing over sliding windows of $500 \mathrm{~ms}$. When rates were stable and low, excitatory STP parameters were modified by a small amount towards facilitation (see Methods and Figs. S2-S4 for variations). For computational reasons we used a total simulation time of 8 hours to model development, but the exact temporal frame does not qualitative change our results (data not shown).

The developmental STP model (dev-STP) maintained a healthy level of firing activity throughout the simulation (i.e. approximately $5 \mathrm{~Hz}$ ) while a tight excitation-inhibition balance in the circuit developed (Fig. 2b). As controls, we 
bioRxiv preprint doi: https://doi.org/10.1101/2021.02.23.431593; this version posted March 12, 2021. The copyright holder for this preprint (which was not certified by peer review) is the author/funder, who has granted bioRxiv a license to display the preprint in perpetuity. It is made available under aCC-BY 4.0 International license.

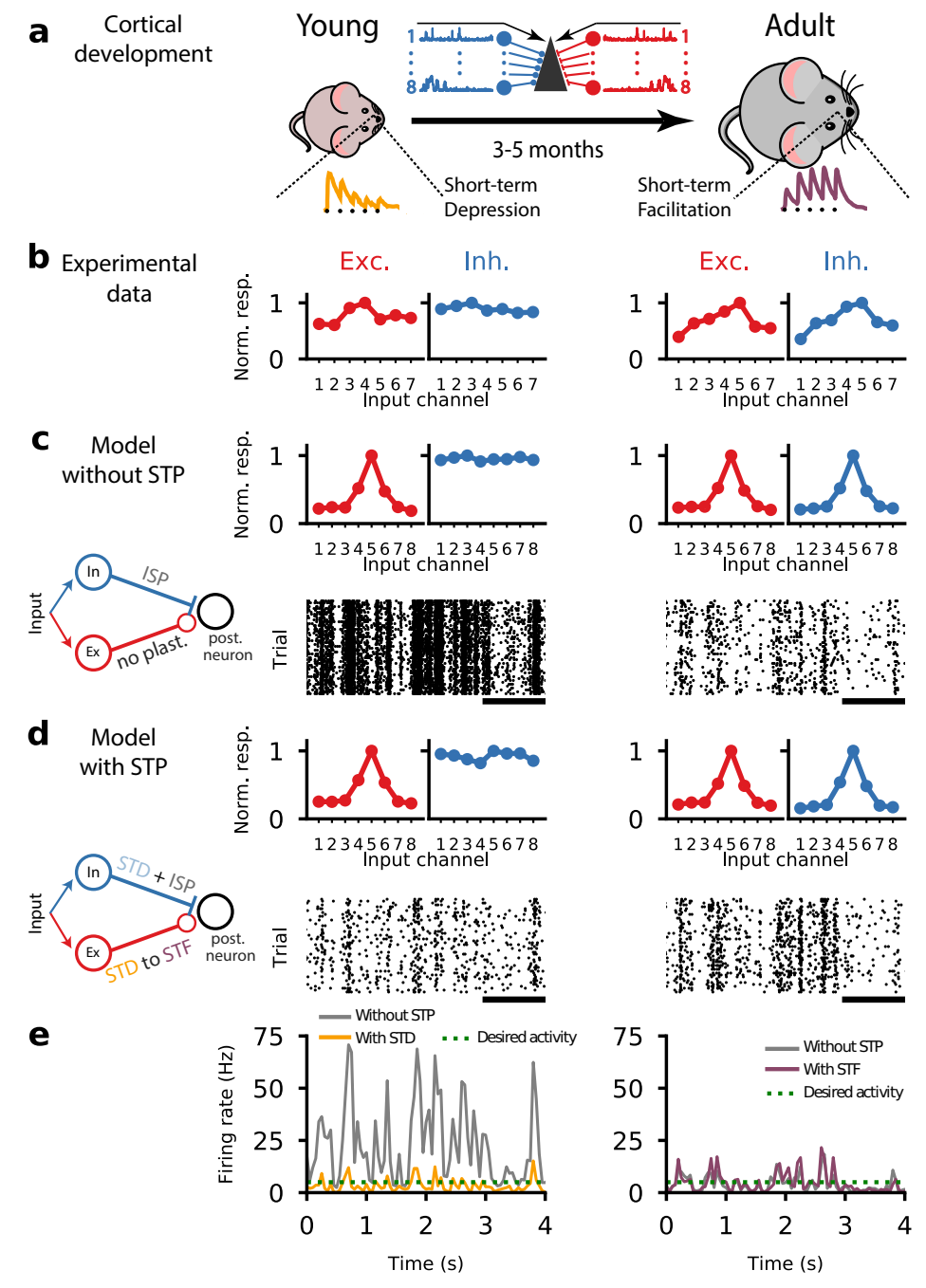

Figure 1. A cortical circuit with short-term synaptic plasticity exhibits healthy neural dynamics in both young and adult conditions. (a) Schematic of animal development from young with short-term depression (left) to adult with short-term facilitation (right) at excitatory synapses as observed experimentally $8-10,13,14$. Traces of short-term synaptic plasticity (STP) for depression (orange) and facilitation (purple) ${ }^{8}$. In the middle is a schematic of the feedforward neural circuit with eight independent input channels, each with an excitatory (red) and an inhibitory (blue) group synapsing onto a postsynaptic neuron (Fig. S1). (b) Inhibitory tuning does not mirror excitatory tuning in young animals (left). Once animals reach adulthood, a precise excitation-inhibition (EI) balance can be observed. Panels adapted from Dorrn et al. ${ }^{25}$. (c) Computational model with long-term synaptic plasticity in inhibitory synapses (ISP; see inset) started from unbalanced excitation-inhibition (top left) and gradually developed El balance (top right). Neuron with unbalanced excitation-inhibition showed high activity ( $\sim 20 \mathrm{~Hz}$; bottom left), which was gradually reduced through ISP ( $4.5 \mathrm{~Hz}$; bottom right). Bottom raster plots represents postsynaptic spiking activity. (d) A computational model with both ISP and STP started from unbalanced excitation-inhibition (top left) and gradually developed El balance (top right). Neuron with unbalanced excitation-inhibition shows low/healthy firing activity $(\sim 4.5 \mathrm{~Hz}$; bottom left) throughout development $(\sim 4.5 \mathrm{~Hz}$; bottom right). Bottom raster plots represents postsynaptic spiking activity. (e) Firing rates of a model without STP (solid gray line) and a model with both ISP and STP in young (left, solid orange line) and adult (right, solid purple line) conditions. Desired activity (dashed green line) represents baseline firing rate as observed experimentally ${ }^{27-30}$.

considered two other models in which STP was fixed either at STD (fixed-STF) or STF (fixed-STF). The fixed-STF scenario exhibited high and more variable firing rates before ISP was able to balance the postsynaptic neuron and lower the firing rates (Fig. 2b,g; Fig. S5). On the other hand, the fixed-STD scenario was able to maintain homeostatic balance throughout the simulation (Fig. 2b,g), but did not develop a tightly balanced inhibitory receptive field (Fig. 2f,h).

Although the developmental STP and fixed-STF models converged to the same mean inhibitory weights (Fig. 2c), the fixed-STF scenario led to substantially higher firing rate variability during development, and large, somewhat 
bioRxiv preprint doi: https://doi.org/10.1101/2021.02.23.431593; this version posted March 12, 2021. The copyright holder for this preprint (which was not certified by peer review) is the author/funder, who has granted bioRxiv a license to display the preprint in perpetuity. It is made available under aCC-BY 4.0 International license.

erratic weight changes (Fig. 2g,d). In contrast dev-STP maintained relatively small weight changes throughout development (Fig. 2d). Finally, while the initial changes of receptive field in the fixed-STF scenario arose quickly, the time of convergence was similar to the dev-STP model (Fig. 2f,i,j), because long-term inhibitory plasticity in the dev-STP scenario sped up dramatically as facilitation developed (Fig. 2b-f). In the dev-STP model, ISP evolved the inhibitory
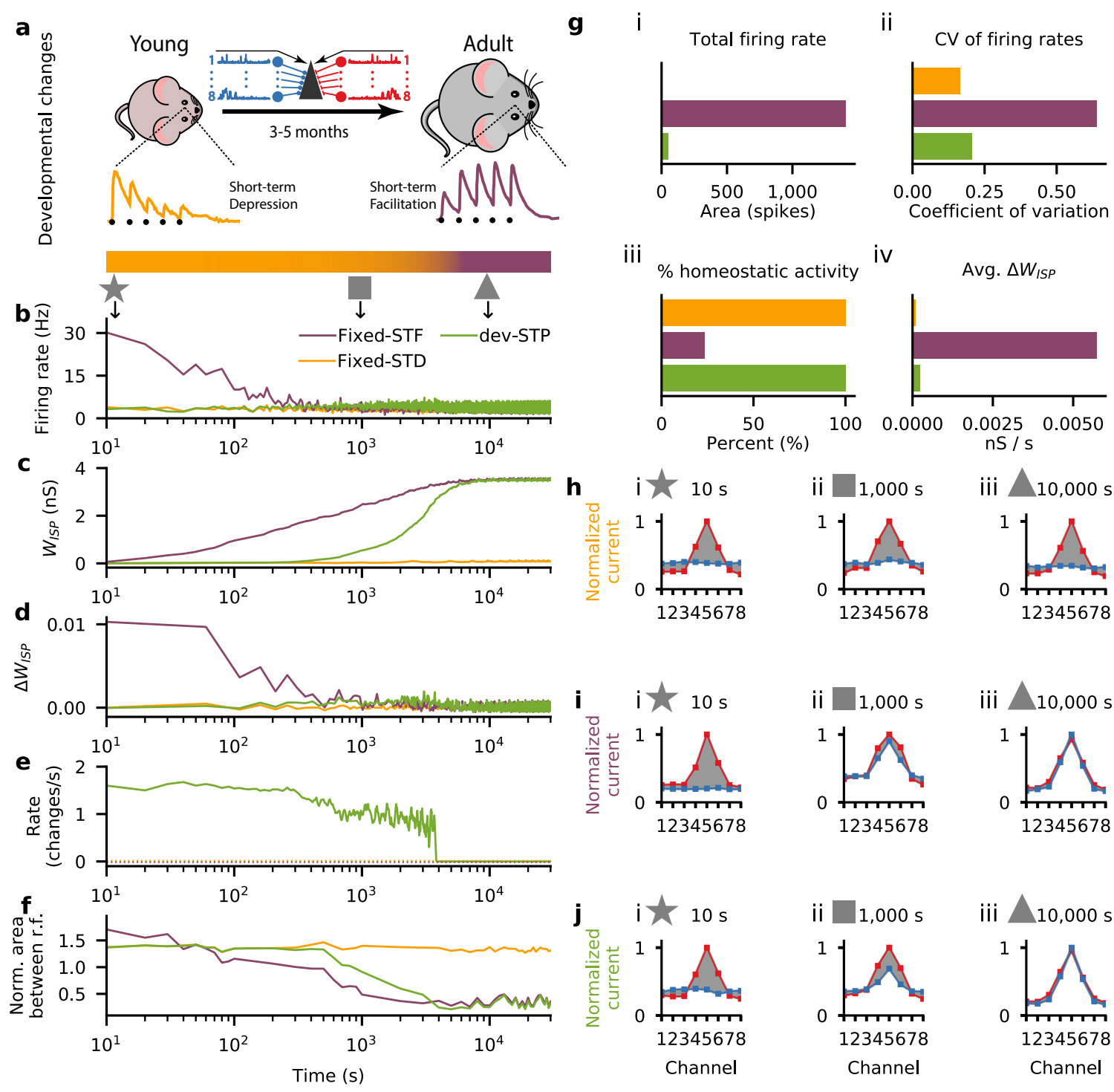

iii $10,000 \mathrm{~s}$
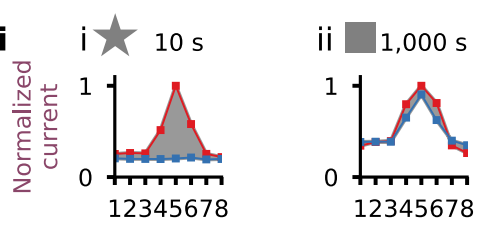

iii $10,000 \mathrm{~s}$
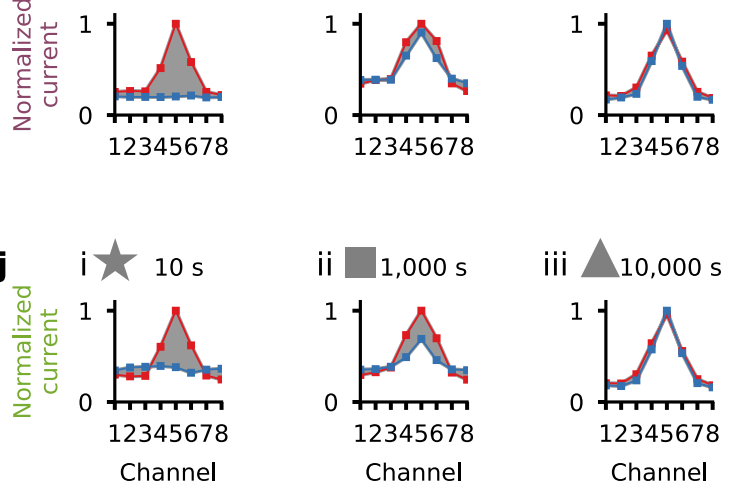

Figure 2. Gradual short-term plasticity shift maintained stable firing rates while detailed El balance developed. (a) Schematic of our developmental short-term plasticity (STP) model (cf. Fig. S1); top: young and adult STP (as in Fig. 1); bottom: gradual changes in STP from depressing to facilitating dynamics (orange and purple respectively, in log-scale as in b-f). (b-f) Different variables of the model across simulated development for three different models: fixed short-term depression (fixed-STD, orange), fixed short-term facilitation (fixed-STF, purple) and developmental model with gradual changes in STP (dev-STP, green line). Note x-axis on log-scale. (b) Receiver neuron firing rate. (c) Mean inhibitory weight. (d) Mean changes in the weight of the inhibitory synaptic afferents. (e) Rate of STP change (note that both fixed-STF and STD remain fixed, shown as dashed lines). (f) Area between normalised excitatory and inhibitory tuning curves (cf. h-j) during the course of simulated development. A normalised area close to 0 represents a perfectly balanced neuron. (g) Additional statistics for the three models. (i) Total neuronal activity calculated using the area between the firing rate in (b) and the desired target rate of $5 \mathrm{~Hz}$. (ii) Average coefficient of variation of the firing rates across simulated development (cf. (b)). (iii) Percent of time spent under homeostasis (i.e. at the desired firing rate; cf. (b)). (iv) Average change in inhibitory weights (cf. (d)). (h-j) Snapshots of excitatory and inhibitory tuning curves across three points in simulated development: 10s (star), 1000s (square) and 10 000s (triangle). Shaded gray area represents difference between excitatory and inhibitory tuning curves (cf. (f)). (h-j) Excitatory (red) and inhibitory (blue) postsynaptic tuning curve for the fixed-STD (h), fixed-STF (i) and dev-STP models (j). 
tuning to match excitation stepwise (Fig. 2f), incrementally handing over control of the target firing rate to inhibition, which ensured postsynaptic activity remained relatively low (Fig. 2b). This means that each increase in the excitatory efficacy through strengthened STF was matched by an increase in the inhibitory efficacy through ISP, until inhibition was fully tuned and the excitatory synapses reach their adult profile of short-term facilitation.
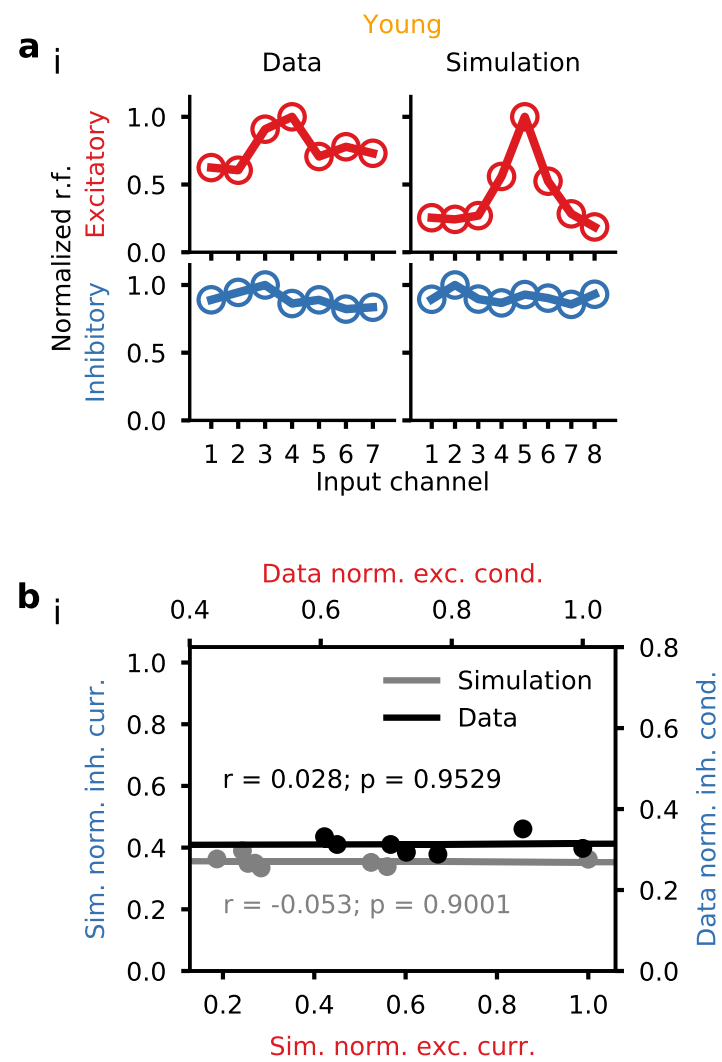

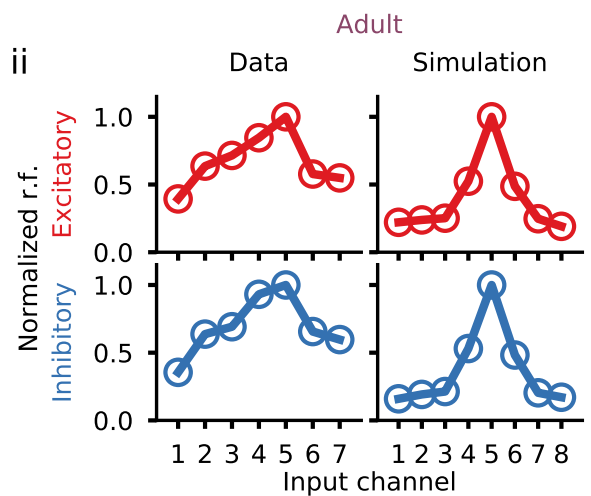

ii

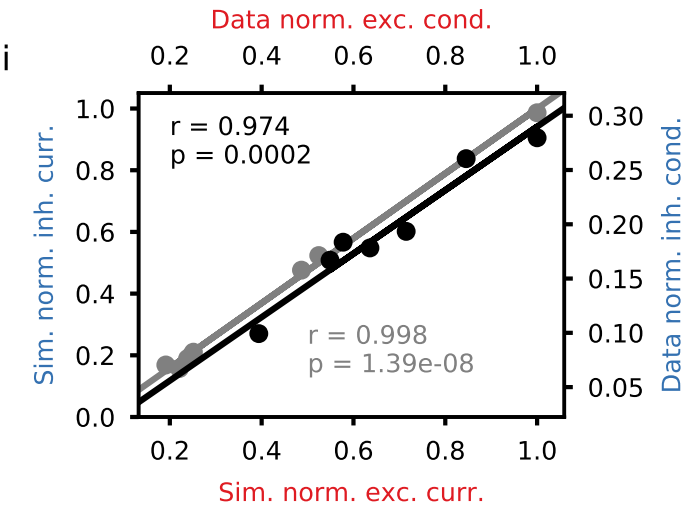

Figure 3. Depression-facilitation shift captured inhibitory receptive field development. (a) Comparison of experimentally observed and simulated (dev-STP model) excitatory and inhibitory tuning curves, for both young (i) and adult (ii) conditions. (b) Excitatory-inhibitory responses for model (gray) and experiments (black). Different dots represent different tone frequencies in the data and different input channels in the model. Lines represent linear correlation between excitatory and inhibitory responses in both model (gray) and experiments (black). Experimental data reproduced from Dorrn et al. ${ }^{25}$.

The dev-STP model was able to maintain the neuron in a (globally) balanced state throughout development while allowing inhibition to gradually mirror the excitatory tuning. In line with experimental in vivo observations in rat auditory cortex across development ${ }^{25}$ inhibitory tuning curves were initially flat (Fig. 3a). In the adult neuron, both model and experiment showed E-I balance. Using the same linear correlation analysis as in the experimental work, we confirmed that excitatory and inhibitory responses in 'young' models were not correlated, but became strongly correlated in the adult profile (Fig. 3b).

\section{Developmental changes in STP shape signal dynamics and transmission}

In line with the establishment of detailed balance ${ }^{21}$, the postsynaptic firing rates in the dev-STP model were initially more correlated with the fixed-STD model, and gradually became more correlated with the fixed-STF model (Fig. 4a,b,c; Fig. S5). Across all input channels we found a gradual decrease of input-output correlation (Fig. 4d)). This was largely due to the fact that the output responses became less correlated with the preferred channel versus the non-preferred channels (Fig. 4e).

Another functional consequence of the changes in short-term dynamics could be observed in the phasic and tonic stimulus responses profiles. Transient (phasic) and steady state (tonic) neural activity has been observed in sensory cortical circuits as part of their stimulus response repertoire ${ }^{27,33,38,39}$. We examined these properties by probing 


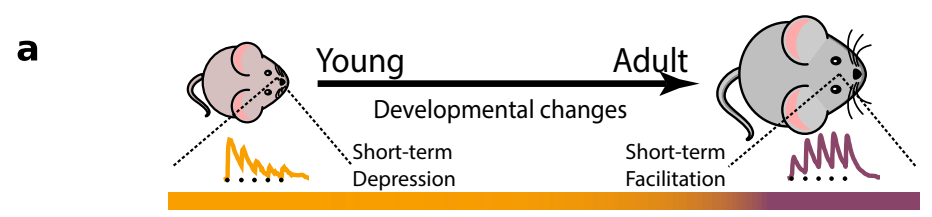

b

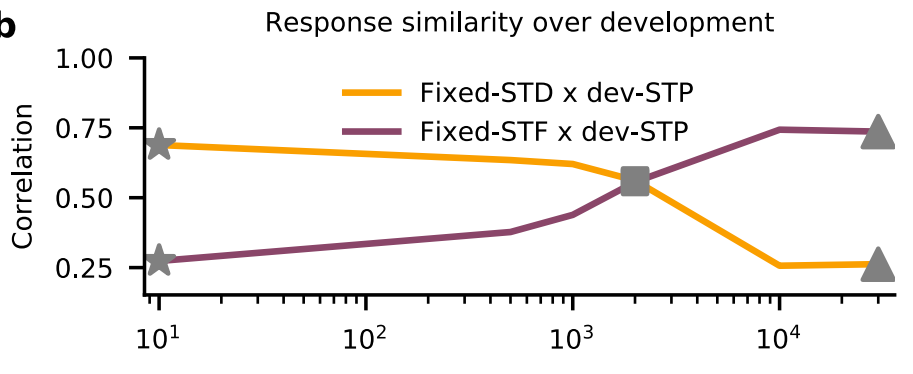

C i

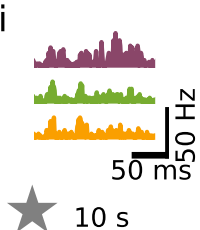

d

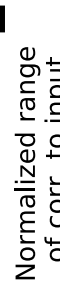

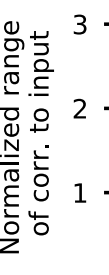

$$
10^{1}
$$

ii

Time (s)

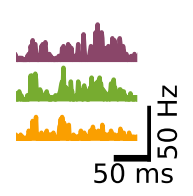

$2,000 \mathrm{~s}$ iii

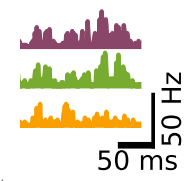

$30,000 \mathrm{~s}$

Input tuning over development

e

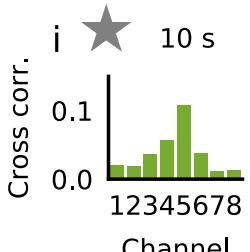

the neuron responses using a step input stimulus (see Methods) (Fig. 5b) to the preferred input channel (channel 5), simulating the sudden presence of a strong sensory feature. We defined the phasic response as the average activity over the first $50 \mathrm{~ms}$ after stimulus onset, and the tonic response as the average rate over the remaining stimulus duration (200ms). Over development, the average phasic activity of the circuit decreased, while the tonic activity increased (Fig. 5b; Fig. S6). These changes in the dynamics are a direct consequence of the gradual change from depressing to facilitating synapses, interacting with the strengthening inhibition. The shift in tonic and phasic responses to a single stimulus also affected subsequent input responses when using two paired step inputs (Fig. 5d inset; Methods). This interaction between subsequent responses was largest for the phasic response, which grew 
a

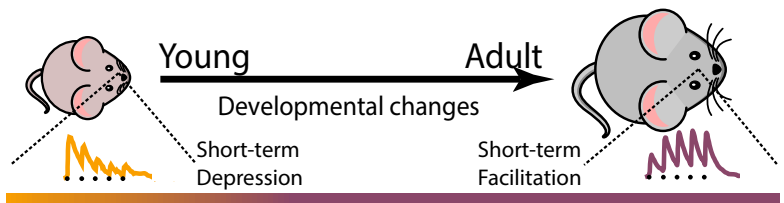

b

Phasic and tonic activities over development

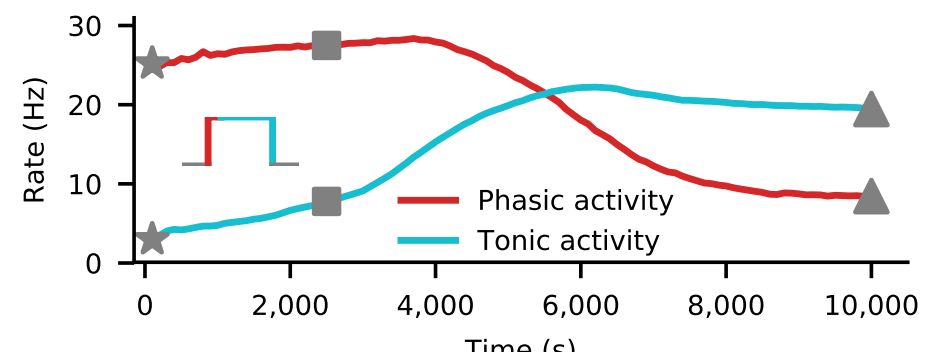

Time (s)

C i

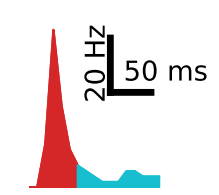

ii

$100 \mathrm{~s}$

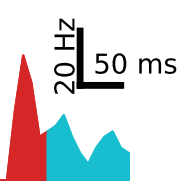

$2,500 \mathrm{~s}$

iii

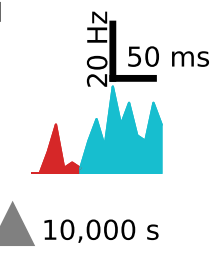

d

Phasic and tonic ratios over development

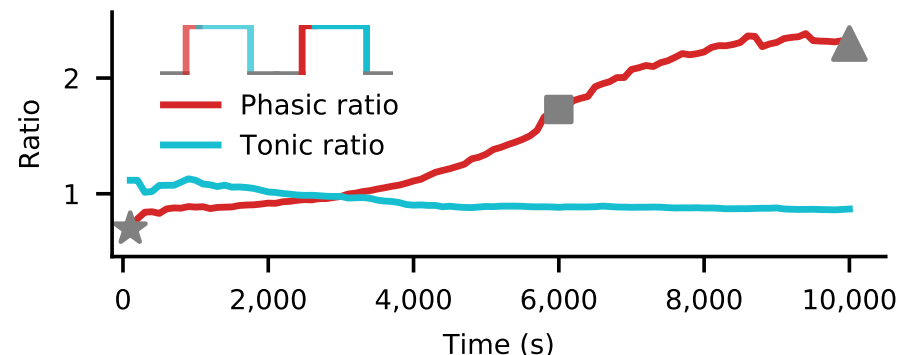

e
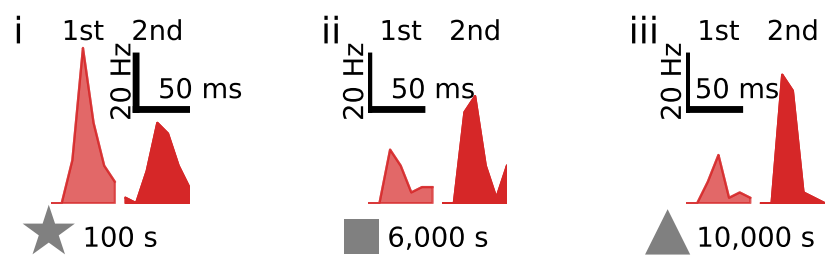

substantially over development, as seen by the increasing ratio of firing rate between the two stimuli (Fig. $5 d, e)$. On the other hand, the tonic response decreased, but only slightly.

We also investigated the phasic response to a step stimulus on very short time scales (Fig. 6a), specifically focusing on the temporal jitter of the first evoked spike (Fig. 6b). In line with previous experimental observations of reduced jitter over development ${ }^{25}$, we observed substantially more stimulus-locked spike times in the adult model than in the young model (Fig. 6c,d). The young scenario showed higher normalized jitter (Methods) than the adult scenario 
$\mathbf{a}_{\mathrm{i}}$

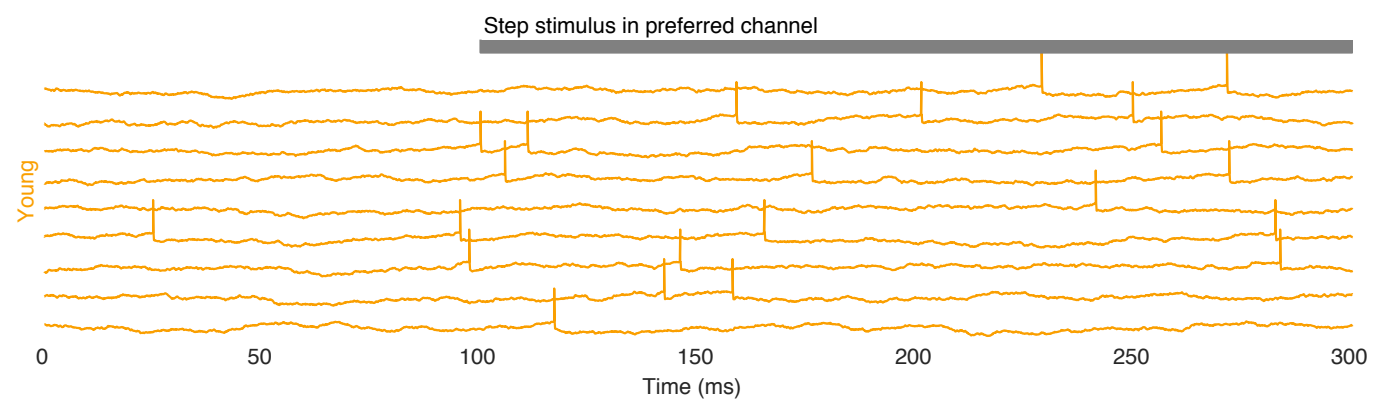

ii

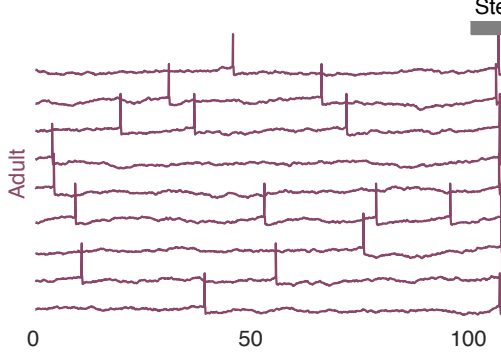

Step stimulus in preferred channel
300

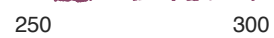

300
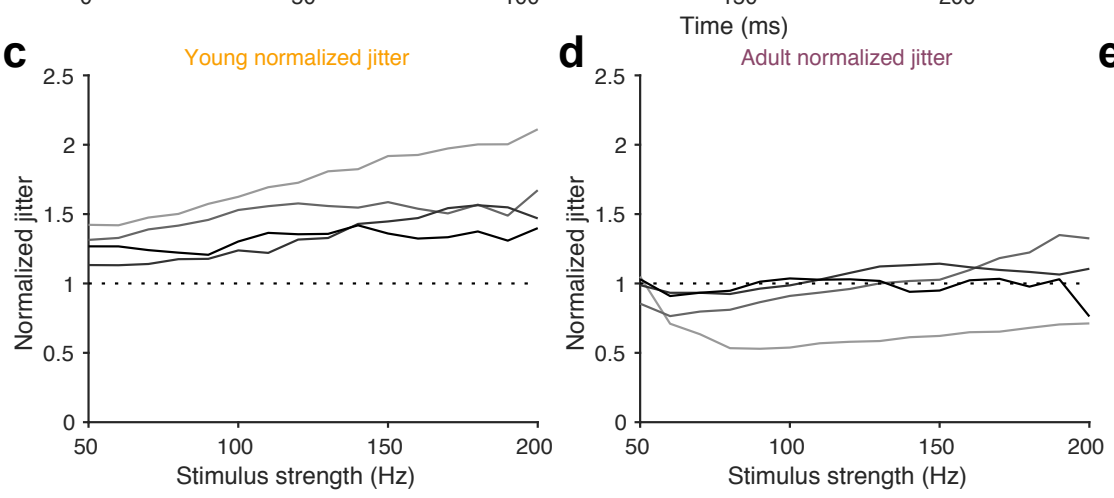

e
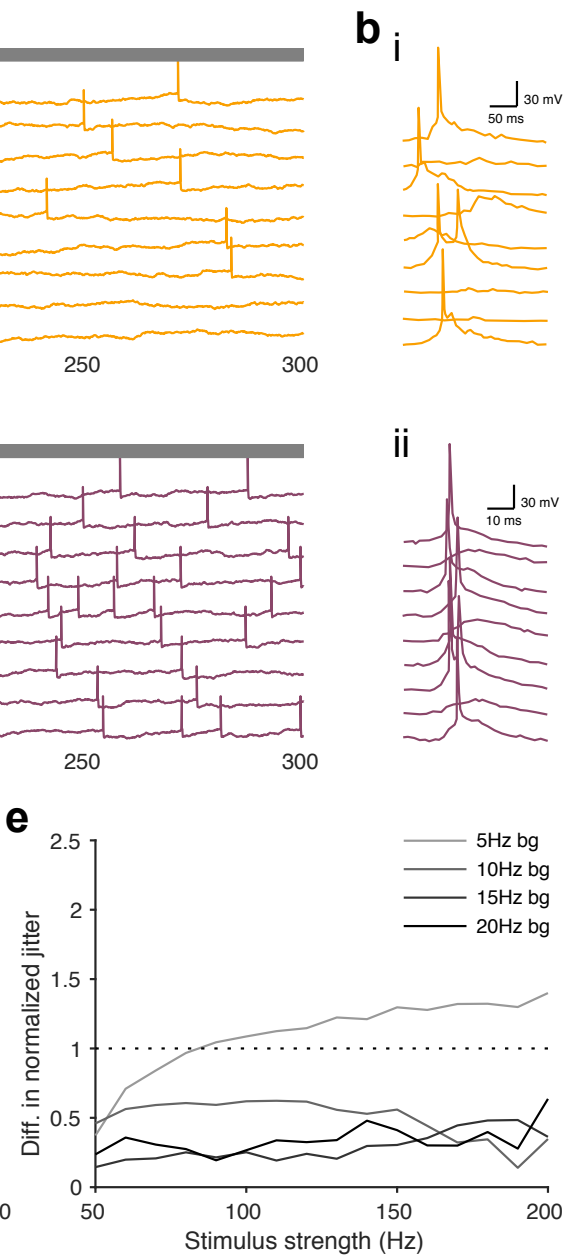

Figure 6. Adult STP improves temporal precision of postsynaptic spikes. (a) Examples of postsynaptic voltage responses with preferred-channel input for both young STP model (i) and adult STP model (ii); gray bar at top represents time during which preferred channel is stimulated. (b) Stimulus evoked responses in in vivo recordings across a few trials in young (i) and adult (ii) animals. Panels adapted from Dorrn et al. ${ }^{25}$. In (a,b) the background firing rate is $5 \mathrm{~Hz}$. (c,d) Normalized jitter of postsynaptic spikes in the young (c) and adult (d) model for different background firing rates (denoted by different shades of gray; see Methods). (e) Difference between normalized jitter of young STP model (c) and adult STP model (d).

across all stimulus strength, and particularly when the background activity before stimulus onset was low (Fig. 6e).

\section{Developmental STP enables working memory properties in a balanced neuron}

Finally, we also investigated the longer term effects of changing STP over development with regard to its implications for short-term memory. Short-term plasticity has recently been proposed as a substrate for working memory ${ }^{40,41}$, owing to the fact that STF can promote increased response to previously displayed stimuli. Here, we tested these ideas in the dev-STP model, by comparing the responses to "recall" stimuli that were or were not preceded by a "preloaded" stimulus.

Models with no STP mechanism, as well as the 'young' dev-STP model showed identical firing rates during the recall period (Fig. 7a,b) independently of whether they had experienced a preloaded stimulus or not. In other words, the 'young' model could not rely on silent working memory traces. The 'adult' dev-STP model, on the other hand, showed substantially higher firing rates during the recall period (Fig. $7 c, d$ ) when the recall stimulus was preceded by a preloaded cue that activated the short-term facilitation in excitatory synapses. Dev-STP thus allowed the neuron to gradually utilise this silent working memory mechanism in a neuron with El balance (Fig. 3a,b, Fig. 7e,f). 
bioRxiv preprint doi: https://doi.org/10.1101/2021.02.23.431593; this version posted March 12, 2021. The copyright holder for this preprint (which was not certified by peer review) is the author/funder, who has granted bioRxiv a license to display the preprint in perpetuity. It is made available under aCC-BY 4.0 International license.
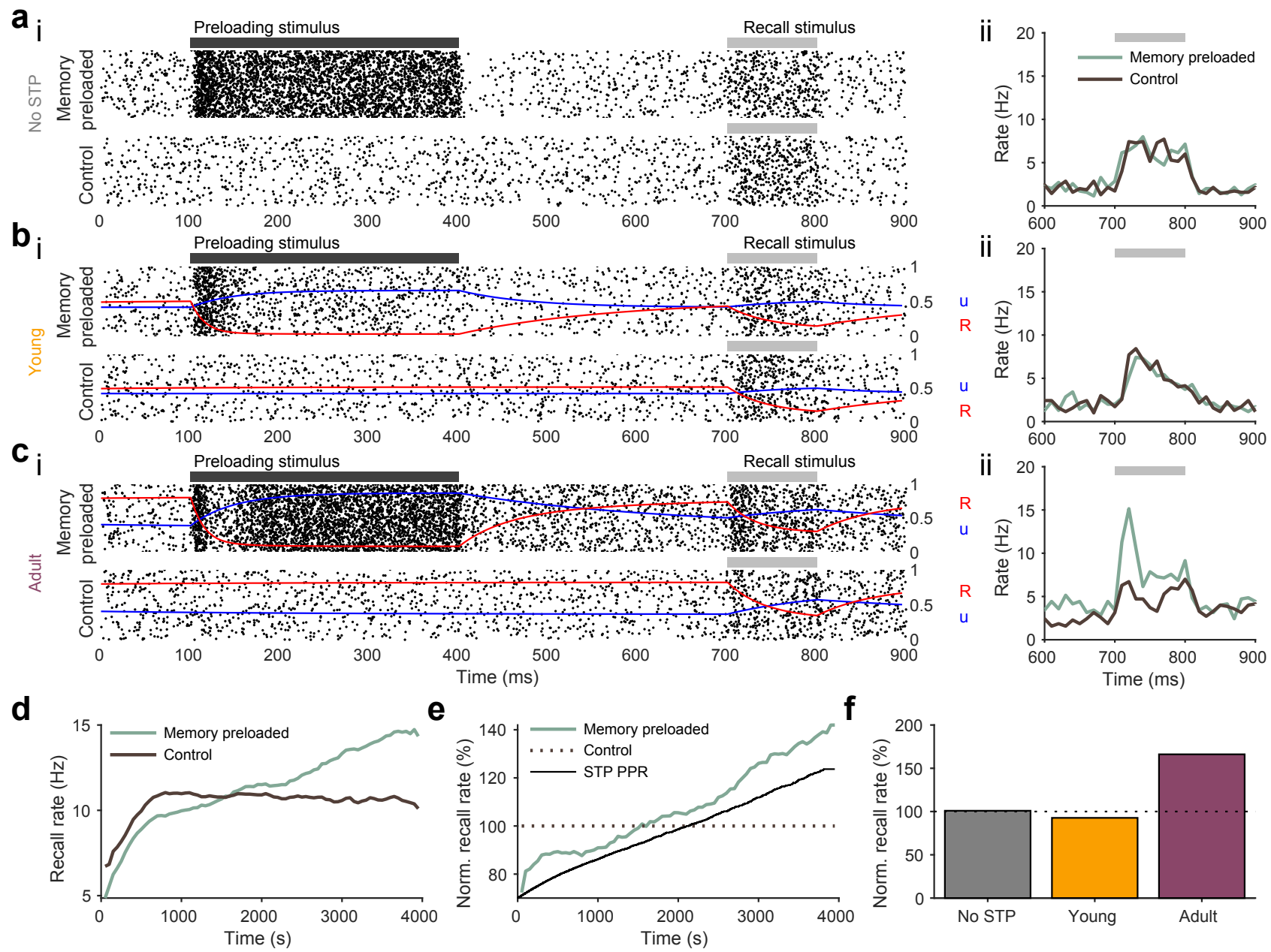

Figure 7. Gradual emergence of synaptic-based working memory over development. (a-c) Raster plot of a working memory test (WMT, i-top)) with a preloaded stimulus and subsequent recall stimulus (black and gray bars respectively) compared with rasterplot of trials without the preloaded stimulus (i-bottom). Average firing rates (ii) for both memory preloaded (light green) and control conditions (dark brown). (a) WMT in a model with only inhibitory synaptic plasticity (i.e. no STP) (b) WMT in a model with young STP profile. (c) WMT in a model with adult STP profile. (d) Firing rates during the recall period with (light green) or without (dark brown) preloaded stimulus. WMTs were preformed every 50 seconds during dev-STP development simulation (cf. Fig. 2) as STP changes from depression to facilitation at excitatory synapses. We only highlight the first $4000 \mathrm{~s}$ of the simulation as after this point STP become minimal. (e) Normalised recall firing rates to the average firing rate of the control case (i.e. without memory preloading). The STP paired-pulse ratio (black) measuring the STP strength of the excitatory synapses for this period is also plotted as reference. (f) Normalized recall rate for three model conditions: no STP (gray), young STP (orange), and adult STP (purple).

\section{Discussion}

It has been widely observed that short-term synaptic dynamics of the cortex change from depressing to facilitating throughout the course of development ${ }^{8-10,12-14,31-35}$. Here, we show that this commonly observed shift in STP may interact with long-term plasticity at inhibitory synapses to form the fundamental architecture of neuronal processing. According to our model, short-term depressing synapses could help to stabilize neural networks in the absence of properly tuned inhibition in young animals (Fig. S5). A gradual change from short-term depression to facilitation then allows for stable dynamics throughout development while inhibitory synaptic plasticity-mediated, detailed excitation-inhibition balance can emerge (Fig. 2). In addition to this stabilising interplay, we show that the developmental maturation of STP also shapes signal processing, by allowing for more temporally precise coding (Fig. 6), and the emergence of synaptic working memory (Fig. 7).

There are currently two dominant views on how changes in STP throughout development may arise. One view is that these changes are caused by sensory experience ${ }^{32}$; the other view poses that these are hard-wired, pre- 
programmed changes ${ }^{13}$. Our developmental STP model suggests a way to reconcile these two views, in that both the sensory-dependent ${ }^{32}$ and non-sensory-dependent ${ }^{13}$ changes observed experimentally may be simply caused by changes in the neural baseline activity. However, although we have modelled changes in STP as a function of neural activity, it is in principle possible to allow for these changes to be purely hard-wired and continuous (cf. Fig. S4). In our hands, the latter mode, i.e. unilateral maturation of STP without heeding the co-development of inhibitory tuning curves, can also lead to stable development (Fig. S4), but this requires fine tuning of a STP change interval, and additional experimental work remains to be done to further study this scenario.

Our work highlights how developmental-STP may shape temporal aspects of synaptic transmission. In particular, our model predicts that young animals primarily encode stimuli with transient, phasic activity, whereas adult animals may transmit both phasic transients and sustained tonic rates equally well. Interestingly, both modes of transmission have been observed in sensory cortices ${ }^{27}$ at different developmental stages. In our model we have assumed that STP changes at all excitatory synapses happen in lockstep over development. However, in the brain not all synapses are modified coincidentally ${ }^{8-10}$, and it is possible that this degree of variability gives a tighter homeostatic control throughout development.

We have focused on long-term inhibitory synaptic plasticity, but excitatory synapses also undergo long-term synaptic plasticity. Importantly, long-term excitatory synaptic plasticity also changes the short-term synaptic dynamics $^{18,42-44}$. It is possible that the gradual changes of STP at excitatory synapses that we have considered here are mediated by long-term excitatory plasticity. In future work it would be interesting to explore the effects of long-term excitatory plasticity with realistic inputs in conjunction with inhibitory synaptic plasticity as a potential model for developmental STP ${ }^{19,36,45}$.

Our model shows a gradual increase in temporal precision of spiking over development, consistent with experimental observations in the auditory cortex of rats ${ }^{25}$, suggesting that STP maturation plays an important role in temporal encoding ${ }^{46-50}$. Our findings add to the growing experimental literature showing that inhibition-excitation balance sharpens spike timings ${ }^{25,49,51,52}$.

Working memory is traditionally thought of as being a property of recurrent neural network dynamics in the prefrontal cortex. However, forms of working memory are also known to exist in sensory cortices ${ }^{53,54}$. Moreover, short-term facilitation has been proposed as a biologically plausible mechanism of working memory at the synaptic level ${ }^{40,55}$. We have shown that working memory-like properties in line with previous theoretical work ${ }^{40}$ gradually emerge in our model as short-term facilitation becomes more dominant. Moreover, we show here that retaining El balance does not interfere with this type of silent working memory. Our results suggest that silent synaptic working memory properties are more likely prevalent in adult cortex, potentially enabling animals to retain information about the recent past even in sensory cortices.

Finally, dysfunctions in the regulation of excitation-inhibition balance underlie numerous neurological disorders ${ }^{56-65}$. In our model we show that short-term plasticity can dynamically control the expression of long-term inhibitory synaptic plasticity, thus modulating E-I balance. Maldaptive developmental STP should thus be reflected in E-I malfunction. Interestingly, this is supported by disease animal models, in which STP and excitation-inhibition balance are both altered in animal models of dysplasia ${ }^{66,67}$.

Overall, our results suggest important functional roles for the commonly observed shift in STP during development.

\section{Acknowledgements}

We would like to thank the Vogels Lab for feedback on an earlier version of this manuscript. D.W.J. was supported by a Marshall Scholarship and a Clarendon Scholarship. R.P.C. and T.P.V. were supported by a Wellcome Trust and Royal Society Sir Henry Dale Fellowship (WT 100000), a Wellcome Trust Senior Research Fellowship (214316/Z/18/Z), and an ERC Consolidator Grant (SYNAPSEEK). 


\section{Supplementary material}

\section{Materials and Methods}

\section{Neuron models}

In this study, we used a conductance-based integrate-and-fire neuron model for simulations ${ }^{68}$. In this model, the membrane voltages are calculated following

$$
\tau \frac{d V}{d t}=-g_{\text {leak }} \cdot\left(V_{\text {rest }}-V\right)+g_{\text {exc }} \cdot\left(E_{\text {exc }}-V\right)+g_{\text {inh }} \cdot\left(E_{\text {inh }}-V\right)
$$

where $V$ is the membrane potential of the neuron as a function of time $t, \tau$ is the membrane time constant, $V_{\text {rest }}$ is the resting membrane potential, $E_{\text {exc }}$ is the excitatory reversal potential, and $E_{\text {inh }}$ is the inhibitory reversal potential. Our neuron parameters are the same as in Vogels and Abbott $^{68}$. In particular, we used a membrane capacitance, $C$, of $200 \mathrm{pF}$ with membrane resistance, $R$, of $100 \mathrm{M} \Omega$, which gives a membrane time constant $\tau=20 \mathrm{~ms}$. $g_{\text {exc }}$ and $g_{\text {inh }}$, expressed in the units of the resting membrane conductance, are the synaptic conductances, and $g_{l}$ is the leaky conductance. The synaptic conductances are modelled as $\tau_{\text {exc }} \frac{d g_{\text {exc }}}{d t}=-g_{\text {ex }}$ and $\tau_{\text {inh }} \frac{d g_{\text {inh }}}{d t}=-g_{\text {in }}$ where $\tau_{\text {exc }}$ and $\tau_{\text {inh }}$ are the synaptic time constants for the excitatory and the inhibitory conductances, respectively. When the neuron receives a presynaptic action potential, its conductance increases by $g_{\text {exc }} \rightarrow g_{\text {exc }}+w_{\text {exc }}$ or $g_{\text {inh }} \rightarrow g_{\text {inh }}+w_{\text {inh }}$ for excitatory and inhibitory synapses, respectively. The model parameters used are summarized in Table 1.

\begin{tabular}{ll}
\hline \hline Parameter & Value \\
\hline$\tau$ & $20.0 \mathrm{~ms}$ \\
$R$ & $100.0 \mathrm{M} \Omega$ \\
$C$ & $200.0 \mathrm{pF}$ \\
$g_{\text {leak }}$ & $10.0 \mathrm{nS}$ \\
$\tau_{\text {exc }}$ & $5.0 \mathrm{~ms}$ \\
$\tau_{\text {inh }}$ & $10.0 \mathrm{~ms}$ \\
$E_{\text {exc }}$ & $0 \mathrm{mV}$ \\
$E_{\text {inh }}$ & $-70 \mathrm{mV}$ \\
$V_{\text {rest }}$ & $-60 \mathrm{mV}$ \\
$V_{\text {thresh }}$ & $-50 \mathrm{mV}$ \\
$V_{\text {reset }}$ & $-60 \mathrm{mV}$ \\
$\tau_{\text {refrac }}$ & $4 \mathrm{~ms}$ \\
$W_{\text {exc }}$ & $0.5 \mathrm{~ns}$ \\
$W_{\text {inh }}$ & $0.1 \mathrm{~ns}$
\end{tabular}

Table 1. Parameter values for conductance-based leaky integrate-and-fire model.

\section{Synaptic plasticity models}

We used both short-term plasticity and long-term inhibitory synaptic plasticity models in our work. Both were calculated separately in the simulations and combined as explained below.

Short-term synaptic plasticity (STP)

Short-term plasticity was used in the simulations following the model defined by ${ }^{5,69,70}$ following

$$
\begin{aligned}
& \frac{d R(t)}{d t}=\frac{1-R(t)}{D}-u(t) R(t) \cdot \delta\left(t-t_{\mathrm{AP}}\right) \\
& \frac{d u(t)}{d t}=\frac{U-u(t)}{F}+f \cdot(1-u(t)) \cdot \delta\left(t-t_{\mathrm{AP}}\right)
\end{aligned}
$$




\begin{tabular}{llllll}
\hline \hline Synaptic dynamics & $D(\mathrm{~s})$ & $F(\mathrm{~s})$ & $U$ & $f$ & $\mathrm{PPR}$ \\
\hline & & & & & \\
Depression & 0.3134 & 0.0798 & 0.3917 & 0.062 & 0.70 \\
Facilitation & 0.0845 & 0.2959 & 0.1973 & 0.1168 & 1.24
\end{tabular}

Table 2. STP parameter values. Paired-pulse ratio (PPR) is given by dividing the second postsynaptic response by the first.

where $R$ models vesicle depletion and $u$ models the presynaptic release probability. Every presynaptic spike at $t_{\mathrm{AP}}$ causes a decrease in $R$, the number of vesicles available by $u R$, which then recovers exponentially to its baseline value of 1 with a time constant $D$. At the same time every presynaptic spike at $t_{A P}$ also causes an increase in the release probability $u$ by $f \cdot(1-u(t))$ (where $f$ is the facilitation rate) and recovers exponentially to its baseline $U$ with a time constant $F$. Finally, the postsynaptic potential, or the weight of the STP component for a synapse exhibiting STP at time $t$ is computed as $w_{\text {STP }}(t)=A R(t) u(t)$, where $A$ is baseline amplitude factor. In simulations, the initial value of $u$ is set to $U$, and the initial value of $R$ is set to 1 . We used the four-parameter version of the TM model $(D, F, U, f)$ as it provides an overall better fit of short-term dynamics data ${ }^{70}$.

\section{STP model fitting}

We found STP parameters which produced excitatory STP paired-pulse responses (PPRs) that matched those found in experiments for young and adult animals. Specifically, we used the STP PPRs observed by Reyes and Sakmann ${ }^{8}$, with excitatory STP PPRs of 0.7 and 1.24 for young and adult animals respectively. In order to find STP parameter values that matched these PPRs, we interpolated between strong STD and strong STF parameter values ${ }^{70}$ (Fig. S1e). Using this interpolation we then calculated the PPR across all parameter sets. We use these PPRs to compared with experimental data from young and adult animals as observed in Reyes and Sakmann ${ }^{8}$. Finally we used least squares to obtain STP parameters that best matched the data in both young (STD) and adult conditions (STF) (see Table 2).

\section{Inhibitory synaptic plasticity}

Long-term inhibitory synaptic plasticity (ISP) is implemented in all inhibitory synapses in all simulations unless otherwise specified. We used the same model as Vogels et al. ${ }^{21}$. In this model, each synapse $i$ has a presynaptic trace $x_{i}$, which increases with each spike by $x_{i} \rightarrow x_{i}+1$ and decays exponentially following $\tau_{\text {STDP }} \frac{d x_{i}}{d t}=-x_{i}$. Then, the synaptic weight of a given synapse following pre- or postsynaptic spikes are updated by

$$
\begin{aligned}
& w_{\text {ISP }} \rightarrow w_{\text {ISP }}+\eta\left(x_{\text {post }}-\alpha\right) \quad \text { with each presynaptic spikes } \\
& w_{\text {ISP }} \rightarrow w_{\text {ISP }}+\eta x_{\text {pre }} \quad \text { with each postsynaptic spikes }
\end{aligned}
$$

where $\eta$ is the learning rate, $\alpha=2 \cdot r_{\text {target }} \cdot \tau_{\mathrm{STDP}}$ is the depression factor, where $\tau_{\mathrm{STDP}}=20 \mathrm{~ms}$ is the STDP time constant, and $r_{\text {target }}=5 \mathrm{~Hz}$ is a constant parameter that defines the target postsynaptic firing rate. In simulations, the initial values of $w_{\text {ISP }}$ is set to zero.

\section{ISP with STP}

In our simulations, ISP is combined with STP in some cases at the inhibitory synapses. In these cases, the total synaptic weight $w_{\text {inh }}$ is computed as the product of the STP and ISP weight components at the time of the postsynaptic spike $w_{\text {inh }}=w_{S T P}^{\text {inh }} \cdot w_{\text {ISP }}$ while the excitatory weight was given by $w_{\text {exc }}=w_{S T P}^{\text {exc }}$.

\section{Simulations}

Input signals and connectivity

To model the neural responses with naturalistic inputs we used 8 independently generated traces of low-pass filtered, half-wave rectified white noise signals. Each of the 8 independent channels represents a signal pathway, and consists of 100 excitatory neurons and 25 inhibitory neurons, giving a total of 1000 presynaptic neurons ${ }^{21}$. All presynaptic neurons synapse onto a single postsynaptic neuron with a total of 1000 synapses, 800 excitatory and 200 inhibitory.

As in Vogels et al. ${ }^{21}$ for each of the 8 channels, we generated its time-varying rates iteratively as $\hat{s}_{k}(t+d t)=$ $\xi-\left(\xi-\hat{s}_{k}(t)\right) \cdot e^{-\frac{d t}{\tau_{s}}}$ where $\hat{s}_{k}$ is the $k$-th signal, $\xi \in[-0.5,0.5]$ is drawn from a uniform distribution, $d t=0.1 \mathrm{~ms}$ is the 
simulation time step, and the filtering time constant is $\tau_{s}=50 \mathrm{~ms}$. We normalized all rates to a preferred firing rate of $100 \mathrm{~Hz}$, and negative values were remove and replaced with a background activity level of $5 \mathrm{~Hz}$.

These traces represent the firing rates across time of each of the 8 input signal channels (see examples in Fig. S1b). We used these rates as seeds to generate Poisson spike trains for each of the eight channels. These inputs were used in the simulations shown in Figures 1, 2, and S5.

\section{Developmental and fixed STP}

When simulating dev-STP, we first found the STP parameters whose paired-pulse ratio (PPR, i.e. EPSP $\left.2 / E_{2} S_{1}\right)$ best matched experimental data ${ }^{8}$. To this end, we started with STP parameters which give strong depression and strong facilitation ${ }^{70}$. Next, we conducted a parameter sweep of the STP parameters from strong depression to strong facilitation using a dense linear space between these two conditions. We then simulated 50 Poisson input spike trains at $35 \mathrm{~Hz}^{8}$, calculated the average PPRs of each train for all STP parameters. We then used the STP parameter values that best matched those of Reyes and Sakmann ${ }^{8}$ for our simulations. These parameter values are summarized in Table 2.

\section{Calibrating the parameters for dev-STP}

Using the STD and STF parameters given in Table 2, we then calculated a set of 3600 parameter values spaced logarithmically between the STD and the STF parameter values. Log interpolation was used instead of linear interpolation because a marginal change towards facilitation generates a higher marginal change in PPR when closer to facilitation than to depression. For each of the 3600 STP parameter values, each time we changed STP parameters, we normalized the STP magnitude parameter $A$ to equal

$$
\begin{aligned}
A & =\frac{w_{\text {baseline }}^{\text {exc }}}{u(t=0) \cdot R(t=0)} \\
& =\frac{w_{\text {baseline }}^{\text {exc }}}{U}
\end{aligned}
$$

where $w_{\text {baseline }}^{\text {exc }}=0.35 \mathrm{nS}$ is the baseline excitatory weight. This normalization fixed the amplitude of the first PSP to the same value, regardless of the STP parameters, thus keeping the baseline weight of excitatory synapses the same throughout development during the simulation (see below for alternative normalizations). Note that the initial value of $u$ is set to $U$, the initial value of $R$ is set to 1 , and the total excitatory weight for a first pre-synaptic spike is given by

$$
\begin{aligned}
w_{\text {exc }}(t=0) & =w_{S T P}^{\text {exc }}(t=0) \\
& =A R(t=0) u(t=0) \\
& =\frac{w_{\text {baseline }}^{\text {exc }}}{U} \cdot 1 \cdot U \\
& =w_{\text {baseline }}^{\text {exc }}
\end{aligned}
$$

regardless of the STP parameters, thus the baseline excitatory weight is invariant across development in our simulations.

To start the dev-STP simulation, we used the baseline STD parameters given in Table 2 at the beginning of the simulation, and slowly changed the parameters from depressing to facilitating at excitatory synapses. Toward this end, we averaged the postsynaptic neuron's firing rate over a 500ms window and monitored how often it exceeded the ISP target rate of $5 \mathrm{~Hz}$ by way of a variable $x_{\text {exceed }}$ that was updated as follows

$$
x_{\text {exceed }}= \begin{cases}x_{\text {exceed }}+\left\lceil\frac{r_{\text {post }}}{r_{\text {target }}}\right] & \text { if } r_{\text {post }} \geq r_{\text {target }} \\ x_{\text {exceed }}-1 & \text { if } r_{\text {post }}<r_{\text {target }}\end{cases}
$$

where $r_{\text {post }}$ is the postsynaptic firing rate and $r_{\text {target }}$ is the ISP target rate (see above). We increment STP to the next set of more facilitating STP parameters when $x_{\text {exceed }} \leq 0$. In other words, the STP parameters are incremented only when the postsynaptic firing rate is equal to or below the ISP target rate for a sufficient period of time, i.e. a time that is proportional to the degree to which the postsynaptic firing rate has exceeded the target rate in the recent past. Changing the excitatory STP to a more facilitating state raises the postsynaptic firing rate, which increases $x_{\text {exceed }}$, thus preventing further facilitating changes in STP until inhibitory synaptic weights strengthen and subsequently decrease 
the postsynaptic firing rate to the target rate, and the cycle starts over. Eventually, the STP parameter values reach the final (experimentally observed ${ }^{8}$ ) STF parameter values (given in Table 2).

For both the fixed-STF and fixed-STD simulations, STP parameters at all excitatory synapses were set to depression and facilitation (Table 2), respectively, for the duration of the simulation.

Further, we quantified the level of "pathological activity" in all three models as the cumulative difference between the observed firing rate and the target firing rate for all input channels (Fig. 2g.i). We also considered the variability of firing rates, i.e. the coefficient of variation (standard deviation divided by the mean) of the firing rates averaged across 10 s bins using a sliding window (Fig. 2g.ii).

\section{Variants of developmental STP model}

We conducted additional simulations to test three variants of the dev-STP model introduced above. In the first control variant we normalized the steady-state PSP amplitudes when using a $5 \mathrm{~Hz}$ presynatic Poisson input (Fig. S2) instead of normalizing to the first PSP. STP parameters in this dev-STP model were modified over development as described above. In this variant, the fixed-STF model displayed a lower initial firing rate than that of the standard model (Fig. S2b), failing to reach the ISP target rate and experimentally observed firing rates in young animals ${ }^{27-30}$. Receptive field development in this variant is otherwise qualitatively similar to our dev-STP model, if somewhat more slowly (Fig. S2g).

In the second control variant we normalized the steady-state PSP of both STD and STF to be equal when using a $10 \mathrm{~Hz}$ (instead of $5 \mathrm{~Hz}$ as in the standard model) presynatic Poisson input (Fig. S3). In this case, STF was weakened enough that fixed-STF in young animals exhibited firing rates near the ISP target rate as observed experimentally ${ }^{27-30}$. However, because of weakened STF, the model failed to develop fine-tuned tuning curves over development (Fig. S3fh).

Finally, we tested a variant of our model in which the developmental shift from STD in young neurons to STF in adult neurons was not activity-dependent. Instead, we altered the dev-STP model to a model in which STP changes occurred at fixed intervals of 3 seconds (Fig. S4e). If these changes occur too frequently, unstable dynamics unfolded so some fine tuning of how often STP changes was required. This third variant also produced qualitatively similar results to our standard dev-STP model (compare Fig. S4, Fig. 2).

\section{Excitatory and inhibitory tuning curves}

To calculate the excitatory and inhibitory tuning curves, we monitored the excitatory and inhibitory conductances for each of the 8 input channels separately, and calculated the respective currents using

$$
\begin{aligned}
& I_{k}^{\text {exc }}(t)=g_{k}^{\text {exc }}(t)\left(E_{\text {exc }}-V(t)\right) \\
& I_{k}^{\text {inh }}(t)=g_{k}^{\text {inh }}(t)\left(E_{\text {inh }}-V(t)\right)+g_{\text {leak }}\left(V_{\text {rest }}-V(t)\right) / K
\end{aligned}
$$

where $l_{k}^{\text {exc }}(t)$ and $l_{k}^{\text {inh }}(t)$ are the excitatory and inhibitory currents and $g_{k}^{\text {exc }}(t)$ and $g_{k}^{\text {inh }}(t)$ are the excitatory and inhibitory conductances of the $k$-th channel at time $t$, respectively ${ }^{21}$. $E_{\text {exc }}$ and $E_{\text {inh }}$ are the excitatory and inhibitory reversal potentials, respectively. $V(t)$ is the postsynaptic membrane potential at time $t$, gleak is the leaky conductance, and $V_{\text {rest }}$ is the resting membrane potential. After calculating the excitatory and inhibitory currents for each channel at all time points, we averaged the excitatory and inhibitory currents across 10 seconds to generate the tuning curves shown in the figures.

\section{Output response dynamics across development}

To measure how the neuron output response changed over the course of simulated development, we stopped the dev-STP simulation (Fig. 2) at 10s, 500s, 1,000s, 2,000s, 10,000s, and 30,000s simulated time and examined the neuron response dynamics of the model. For each snapshot, we ran 50 step current trials with frozen parameters and compared the average firing rates of the dev-STP scenario with those of the fixed-STD and fixed-STF scenarios (Fig. 4b).

To investigate how input tuning changed over development, we calculated the cross correlations between the input and output rates for each of the 8 channels ${ }^{21}$. We obtained the correlation range by subtracting the minimum from the maximum correlation and normalized the range by dividing by the mean correlation of all channels with the output (Fig. 4d). 


\section{Signal transmission across development}

To investigate signal transmission across development, we presented a $250 \mathrm{~ms}$ long $150 \mathrm{~Hz}$ input stimulus to the preferred input channel every 100 seconds of the dev-STP simulation (Fig. 2). We analysed the output firing rates during the first $50 \mathrm{~ms}$ after stimulus onset (phasic period) and the remaining $200 \mathrm{~ms}$ afterwards (tonic period); Fig. 5b,c). We also tested a double step input stimulus, two $250 \mathrm{~ms} 150 \mathrm{~Hz}$ input stimuli separated by $250 \mathrm{~ms}$ of spontaneous activity (Fig. 5d,e).

\section{Temporal precision simulations}

We compared the temporal precision of postsynaptic spikes in our model with experimental observations ${ }^{25}$. To this end, we stimulated the preferred channel (5) of the output neuron with a $200 \mathrm{~ms}$ step current, imitating a pure tone in the preferred frequency in the auditory cortex ${ }^{25}$. To quantify the temporal precision of the response, we calculated the standard deviation of the delay between the stimulus onset and the first postsynaptic spike, denoted as the jitter ${ }^{25}$. To allow comparison across different firing rates, we also calculated a normalized jitter, i.e., the jitter's coefficient of variation. The normalized jitter was compared for different preferred-channel stimulus strengths as well as for varying spontaneous activity levels (Fig. 6c-e).

\section{Working memory}

To test for working memory-like properties, we used two simulation protocols. In the "memory preloaded" trials, we stimulated the neuron with a $300 \mathrm{~ms}$ long $150 \mathrm{~Hz}$ steady state stimulus (a memory) in the preferred channel. All remaining channels received spontaneous rates at $5 \mathrm{~Hz}$. After the memory preloading period, the preferred channel input received spontaneous firing rate inputs for a $300 \mathrm{~ms}$ delay period, followed by a weaker, $100 \mathrm{~ms}$ long $50 \mathrm{~Hz}$ "recall cue" stimulus. For "control" trials, the input channels of the neuron only received the $100 \mathrm{~ms}$ recall cue, to the preferred channel, without preloading.

We then compared the firing rates during recall between the memory preloaded and control trials, to study the 'silent' working memory effects in our model. We tested this throughout simulated development, by freezing the dev-STP simulation every 50s and simulating 500 trials of the memory-preloaded simulations and 500 trials of the control simulations.

Simulator

Simulations were conducted in Python using Brian Simulator 2. Code to reproduce our key findings is available at github.com/djia/dev-stp. 


\section{References}

[1] R. S. Zucker and W. G. Regehr. Short-term synaptic plasticity. Annu Rev Physiol, 64(1):355-405, January 2002.

[2] L. F. Abbott and W. Regehr. Synaptic computation. Nature, 431(7010):796-803, 2004.

[3] W. G. Regehr. Short-Term Presynaptic Plasticity. Cold Spring Harbor Perspectives in Biology, 4(7):a005702-a005702, July 2012.

[4] A. Reyes, R. Lujan, A. Rozov, N. Burnashev, P. Somogyi, and B. Sakmann. Target-cell-specific facilitation and depression in neocortical circuits. Nature neuroscience, 1(4):279-285, 1998.

[5] H. Markram, Y. Wang, and M. Tsodyks. Differential signaling via the same axon of neocortical pyramidal neurons. Proc. Natl. Acad. Sci. USA, 95(9):5323-5328, 1998.

[6] A. E. Takesian, V. C. Kotak, and D. H. Sanes. Presynaptic GABAB receptors regulate experience-dependent development of inhibitory short-term plasticity. The Journal of Neuroscience, 30(7):2716-2727, 2010.

[7] A. Reyes. Synaptic short-term plasticity in auditory cortical circuits. Hearing research, 2011.

[8] A. Reyes and B. Sakmann. Developmental switch in the short-term modification of unitary epsps evoked in layer 2/3 and layer 5 pyramidal neurons of rat neocortex. Journal of Neuroscience, 19(10):3827-3835, 1999.

[9] Z. Zhang. Maturation of layer $\mathrm{V}$ pyramidal neurons in the rat prefrontal cortex: intrinsic properties and synaptic function. Journal of Neurophysiology, (91):1171-1182, 2004.

[10] A.-M. M. Oswald and A. D. Reyes. Maturation of intrinsic and synaptic properties of layer 2/3 pyramidal neurons in mouse auditory cortex. Journal of Neurophysiology, 99(6):2998-3008, June 2008.

[11] S. Dasari and Y. Yuan. Low level postnatal methylmercury exposure in vivo alters developmental forms of short-term synaptic plasticity in the visual cortex of rat. Toxicology and applied pharmacology, 240(3):412-422, 2009.

[12] C. E. J. Cheetham and K. Fox. Presynaptic development at $L 4$ to $12 / 3$ excitatory synapses follows different time courses in visual and somatosensory cortex. The Journal of neuroscience : the official journal of the Society for Neuroscience, 30(38):12566-12571, September 2010.

[13] W. X. Chen and D. V. Buonomano. Developmental shift of short-term synaptic plasticity in cortical organotypic slices. Neuroscience, 213:38-46, June 2012.

[14] M. I. Kerr, M. J. Wall, and M. J. E. Richardson. Adenosine A1 receptor activation mediates the developmental shift at layer 5 pyramidal cell synapses and is a determinant of mature synaptic strength. The Journal of physiology, 591(Pt 13):3371-3380, July 2013.

[15] H. Ko, S. B. Hofer, B. Pichler, K. A. Buchanan, P. J. Sjostrom, and T. D. Mrsic-Flogel. Functional specificity of local synaptic connections in neocortical networks. Nature, 473(7345):87-91, April 2011.

[16] M. Tsodyks and H. Markram. The neural code between neocortical pyramidal neurons depends on neurotransmitter release probability. Proc. Natl. Acad. Sci. USA, 94(2):719-723, 1997.

[17] D. Sussillo, T. Toyoizumi, and W. Maass. Self-tuning of neural circuits through short-term synaptic plasticity. Journal of Neurophysiology, 97(6):4079-4095, June 2007.

[18] R. P. Costa, B. E. P. Mizusaki, P. J. Sjostrom, and M. C. W. van Rossum. Functional consequences of pre- and postsynaptic expression of synaptic plasticity. Philosophical transactions of the Royal Society of London. Series B, Biological sciences, 372(1715): 20160153, March 2017.

[19] J. A. D'amour and R. C. Froemke. Inhibitory and excitatory spike-timing-dependent plasticity in the auditory cortex. Neuron, 86 (2):514-528, 2015.

[20] R. C. Froemke. Plasticity of Cortical Excitatory-Inhibitory Balance. Annual Review of Neuroscience, 38(1):150421150146009, April 2015.

[21] T. P. Vogels, H. Sprekeler, F. Zenke, C. Clopath, and W. Gerstner. Inhibitory plasticity balances excitation and inhibition in sensory pathways and memory networks. Science, 334(6062):1569-1573, 2011.

[22] T. P. Vogels, R. C. Froemke, N. Doyon, M. Gilson, J. S. Haas, R. Liu, A. Maffei, P. Miller, C. Wierenga, M. A. Woodin, et al. Inhibitory synaptic plasticity: spike timing-dependence and putative network function. Frontiers in neural circuits, 7:119, 2013.

[23] G. Hennequin, E. J. Agnes, and T. P. Vogels. Inhibitory plasticity: balance, control, and codependence. Annual Review of Neuroscience, 40:557-579, 2017. 
[24] R. C. Froemke, M. M. Merzenich, and C. E. Schreiner. A synaptic memory trace for cortical receptive field plasticity. Nature, 450 (7168):425, 2007.

[25] A. L. Dorrn, K. Yuan, A. J. Barker, C. E. Schreiner, and R. C. Froemke. Developmental sensory experience balances cortical excitation and inhibition. Nature, 465(7300):932-936, 2010

[26] A. Marín-Burgin, L. A. Mongiat, M. B. Pardi, and A. F. Schinder. Unique processing during a period of high excitation/inhibition balance in adult-born neurons. Science, 335(6073):1238-1242, 2012.

[27] S. Sakata and K. D. Harris. Laminar structure of spontaneous and sensory-evoked population activity in auditory cortex. Neuron, 64(3):404-418, 2009.

[28] C. M. Niell and M. P. Stryker. Highly selective receptive fields in mouse visual cortex. Journal of Neuroscience, 28(30):7520-7536, 2008.

[29] P. Charlesworth, E. Cotterill, A. Morton, S. G. Grant, and S. J. Eglen. Quantitative differences in developmental profiles of spontaneous activity in cortical and hippocampal cultures. Neural development, 10(1):1, 2015.

[30] E. Cotterill, D. Hall, K. Wallace, W. R. Mundy, S. J. Eglen, and T. J. Shafer. Characterization of early cortical neural network development in multiwell microelectrode array plates. Journal of biomolecular screening, 21(5):510-519, 2016.

[31] F. Jia, H. Wei, X. Li, X. Xie, and Y. Zhou. Short-term synaptic plasticity in the rat geniculo-cortical pathway during development in vivo. Neuroscience letters, 398(1-2):73-77, 2006.

[32] C. Cheetham and K. Fox. The role of sensory experience in presynaptic development is cortical area-specific. The Journal of physiology, 2011.

[33] A. Frick, D. Feldmeyer, and B. Sakmann. Postnatal development of synaptic transmission in local networks of L5A pyramidal neurons in rat somatosensory cortex. The Journal of physiology, 585(1):103-116, November 2007.

[34] S. J. Etherington and S. R. Williams. Postnatal development of intrinsic and synaptic properties transforms signaling in the layer 5 excitatory neural network of the visual cortex. Journal of Neuroscience, 31(26):9526-9537, 2011.

[35] P. Wasling. Developmental Changes in Release Properties of the CA3-CA1 Glutamate Synapse in Rat Hippocampus. Journal of Neurophysiology, 92(5):2714-2724, July 2004.

[36] C. Clopath, T. P. Vogels, R. C. Froemke, and H. Sprekeler. Receptive field formation by interacting excitatory and inhibitory synaptic plasticity. bioRxiv, page 066589, 2016.

[37] S. Ruder. An overview of gradient descent optimization algorithms. arXiv preprint arXiv:1609.04747, 2016.

[38] A. Luczak, B. L. McNaughton, and K. D. Harris. Packet-based communication in the cortex. Nature Reviews Neuroscience, 16(12): 745, 2015.

[39] P. Bartho, C. Curto, A. Luczak, S. L. Marguet, and K. D. Harris. Population coding of tone stimuli in auditory cortex: dynamic rate vector analysis. European Journal of Neuroscience, 30(9):1767-1778, 2009.

[40] G. Mongillo, O. Barak, and M. Tsodyks. Synaptic theory of working memory. Science, 319(5869):1543-1546, 2008.

[41] D. Hansel and G. Mato. Short-term plasticity explains irregular persistent activity in working memory tasks. The Journal of neuroscience : the official journal of the Society for Neuroscience, 33(1):133-149, January 2013.

[42] H. Markram and M. Tsodyks. Redistribution of synaptic efficacy between neocortical pyramidal neurons. Nature, 1996.

[43] P. J. Sjöström, G. G. Turrigiano, and S. Nelson. Multiple forms of long-term plasticity at unitary neocortical layer 5 synapses. Neuropharmacology, 52(1):176-184, 2007.

[44] R. P. Costa, R. C. Froemke, P. J. Sjostrom, and M. C. W. van Rossum. Unified pre- and postsynaptic long-term plasticity enables reliable and flexible learning. eLife, 4:e09457, 2015.

[45] L. Wang and A. Maffei. Inhibitory plasticity dictates the sign of plasticity at excitatory synapses. Journal of Neuroscience, 34(4): 1083-1093, January 2014. doi: 10.1523/jneurosci.4711-13.2014. URL https://doi.org/10.1523/jneurosci.4711-13.2014.

[46] M. Abeles, H. Bergman, E. Margalit, and E. Vaadia. Spatiotemporal firing patterns in the frontal cortex of behaving monkeys. Journal of neurophysiology, 70(4):1629-1638, 1993.

[47] A. Riehle, S. Grün, M. Diesmann, and A. Aertsen. Spike synchronization and rate modulation differentially involved in motor cortical function. Science, 278(5345):1950-1953, 1997. 
[48] S. Panzeri, R. S. Petersen, S. R. Schultz, M. Lebedev, and M. E. Diamond. The role of spike timing in the coding of stimulus location in rat somatosensory cortex. Neuron, 29(3):769-777, 2001.

[49] M. Wehr and A. M. Zador. Balanced inhibition underlies tuning and sharpens spike timing in auditory cortex. Nature, 426(6965): 442, 2003.

[50] M. A. Montemurro, S. Panzeri, M. Maravall, A. Alenda, M. R. Bale, M. Brambilla, and R. S. Petersen. Role of precise spike timing in coding of dynamic vibrissa stimuli in somatosensory thalamus. Journal of neurophysiology, 98(4):1871-1882, 2007.

[51] F. Pouille and M. Scanziani. Enforcement of temporal fidelity in pyramidal cells by somatic feed-forward inhibition. Science, 293 (5532):1159-1163, 2001.

[52] J. Kremkow, A. Aertsen, and A. Kumar. Gating of signal propagation in spiking neural networks by balanced and correlated excitation and inhibition. The Journal of neuroscience, 30(47):15760-15768, 2010.

[53] T. Pasternak and M. W. Greenlee. Working memory in primate sensory systems. Nature Reviews Neuroscience, 6(2):97, 2005.

[54] T. B. Christophel, P. C. Klink, B. Spitzer, P. R. Roelfsema, and J.-D. Haynes. The distributed nature of working memory. Trends in Cognitive Sciences, 21(2):111-124, February 2017. doi: 10.1016/j.tics.2016.12.007. URL https://doi.org/10.1016/j.tics.2016.12.007.

[55] M. G. Stokes. 'activity-silent' working memory in prefrontal cortex: a dynamic coding framework. Trends in Cognitive Sciences, 19(7):394-405, July 2015. doi: 10.1016/j.tics.2015.05.004. URL https://doi.org/10.1016/j.tics.2015.05.004.

[56] S. A. Eichler and J. C. Meier. Ei balance and human diseases-from molecules to networking. Frontiers in molecular neuroscience, $1: 2,2008$.

[57] K. Gale. Gaba and epilepsy: basic concepts from preclinical research. Epilepsia, 33:S3-12, 1992.

[58] H. Bradford. Glutamate, gaba and epilepsy. Progress in neurobiology, 47(6):477-511, 1995.

[59] S. M. Thompson, C. Fortunato, R. A. McKinney, M. Müller, and B. H. Gähwiler. Mechanisms underlying the neuropathological consequences of epileptic activity in the rat hippocampus in vitro. Journal of Comparative Neurology, 372(4):515-528, 1996.

[60] R. W. Olsen and M. Avoli. Gaba and epileptogenesis. Epilepsia, 38(4):399-407, 1997.

[61] I. Cobos, M. E. Calcagnotto, A. J. Vilaythong, M. T. Thwin, J. L. Noebels, S. C. Baraban, and J. L. Rubenstein. Mice lacking dlx1 show subtype-specific loss of interneurons, reduced inhibition and epilepsy. Nature neuroscience, 8(8):1059, 2005.

[62] D. A. Lewis, L. A. Glantz, J. N. Pieppi, and R. A. Sweet. Altered cortical glutamate neurotransmission in schizophrenia: evidence from morphological studies of pyramidal neurons. Annals of the New York Academy of Sciences, 1003(1):102-112, 2003.

[63] C. Kehrer, N. Maziashvili, T. Dugladze, and T. Gloveli. Altered excitatory-inhibitory balance in the nmda-hypofunction model of schizophrenia. Frontiers in molecular neuroscience, 1:6, 2008.

[64] S. Jamain, H. Quach, C. Betancur, M. Råstam, C. Colineaux, I. C. Gillberg, H. Soderstrom, B. Giros, M. Leboyer, C. Gillberg, et al. Mutations of the x-linked genes encoding neuroligins nlgn3 and nlgn4 are associated with autism. Nature genetics, 34(1):27, 2003.

[65] F. Laumonnier, F. Bonnet-Brilhault, M. Gomot, R. Blanc, A. David, M.-P. Moizard, M. Raynaud, N. Ronce, E. Lemonnier, P. Calvas, et al. X-linked mental retardation and autism are associated with a mutation in the nlgn4 gene, a member of the neuroligin family. The American Journal of Human Genetics, 74(3):552-557, 2004.

[66] H.-X. Chen, H. Xiang, and S. N. Roper. Impaired developmental switch of short-term plasticity in pyramidal cells of dysplastic cortex. Epilepsia, 48(1):141-148, 2007.

[67] F.-W. Zhou, H.-X. Chen, and S. N. Roper. Balance of inhibitory and excitatory synaptic activity is altered in fast-spiking interneurons in experimental cortical dysplasia. Journal of neurophysiology, 102(4):2514-2525, 2009.

[68] T. P. Vogels and L. F. Abbott. Signal Propagation and Logic Gating in Networks of Integrate-and-Fire Neurons. The Journal of neuroscience : the official journal of the Society for Neuroscience, 25(46):10786-10795, November 2005.

[69] M. Tsodyks, K. Pawelzik, and H. Markram. Neural Networks with Dynamic Synapses. Neural Computation, 1998.

[70] R. P. Costa, P. J. Sjostrom, and M. C. W. van Rossum. Probabilistic inference of short-term synaptic plasticity in neocortical microcircuits. Frontiers in computational neuroscience, 7:75, 2013. 
bioRxiv preprint doi: https://doi.org/10.1101/2021.02.23.431593; this version posted March 12, 2021. The copyright holder for this preprint (which was not certified by peer review) is the author/funder, who has granted bioRxiv a license to display the preprint in perpetuity. It is made available under aCC-BY 4.0 International license.

493

\section{Supplementary figures}

a
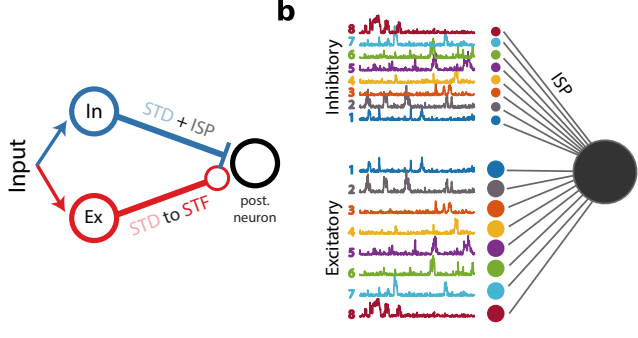

C

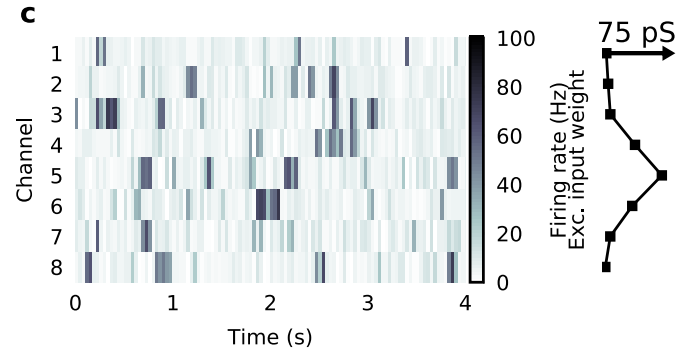

d

STP parameters for dev-STP

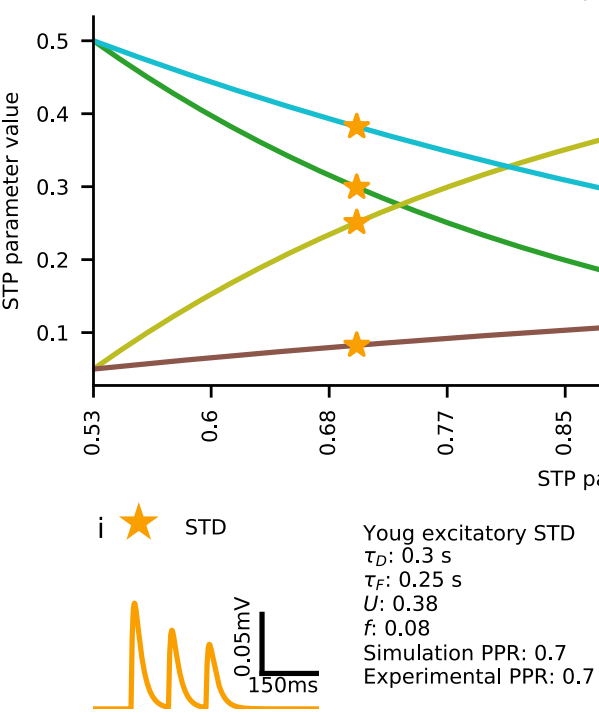

Figure S1. Details of the cortical circuit and plasticity models. (a) Schematic of a single channel feedforward circuit with correlated excitatory and inhibitory input, and the respective forms of plasticity. (b) Feedforward neural circuit with 8 channels and correlated excitatory and inhibitory inputs. (c) Left: example of input given to the 8 channel feedforward neural circuit; right: excitatory tuning curve strength for each of the 8 channels. (d) Each of the four STP parameters, $\tau_{D}, \tau_{F}, U$, and $f$ resulting in different paired-pulse ratios (PPRs) (Table 2). Parameters matching the young (orange star) and adult (purple triangle) STP PPRs as used in the dev-STP model are highlighted. (e) Example postsynaptic potential traces for the STP parameter values of both young (i) and adult animals (ii; cf. d). 
bioRxiv preprint doi: https://doi.org/10.1101/2021.02.23.431593; this version posted March 12, 2021. The copyright holder for this preprint (which was not certified by peer review) is the author/funder, who has granted bioRxiv a license to display the preprint in perpetuity. It is made available under aCC-BY 4.0 International license.
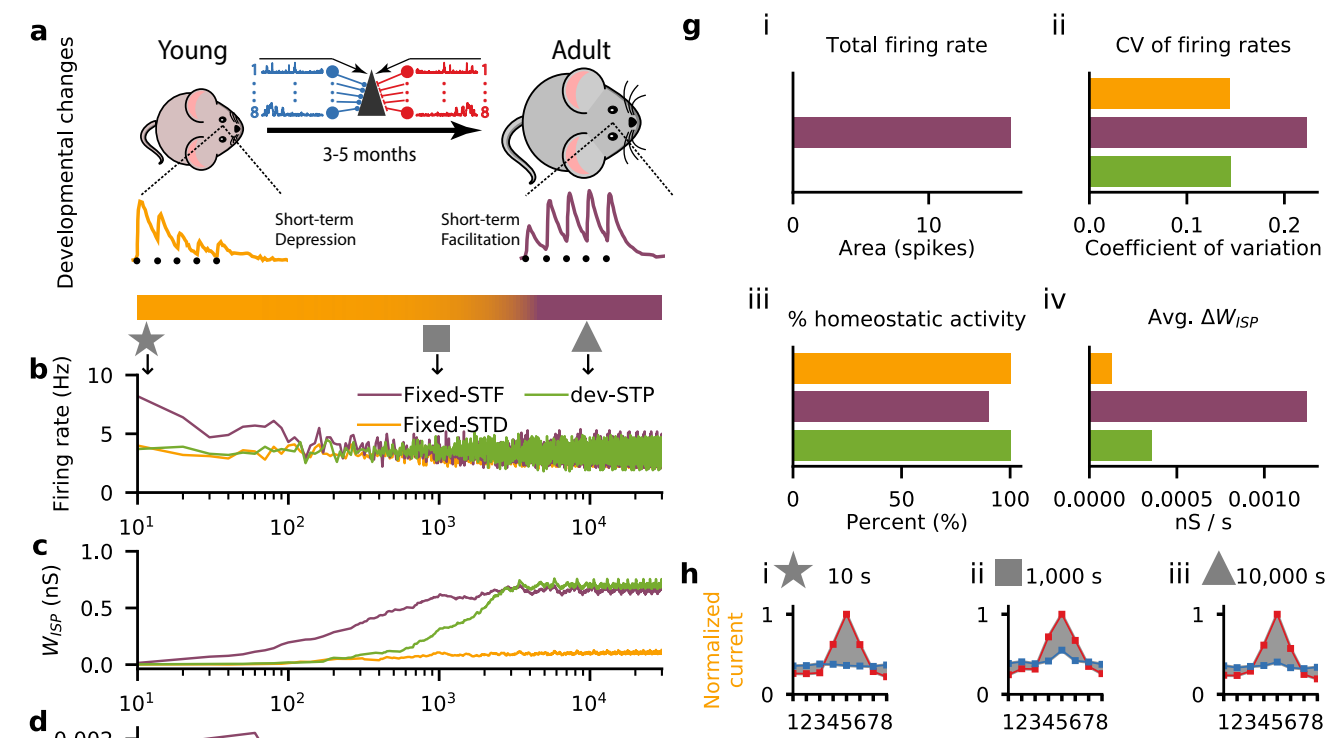

iii $10,000 \mathrm{~s}$
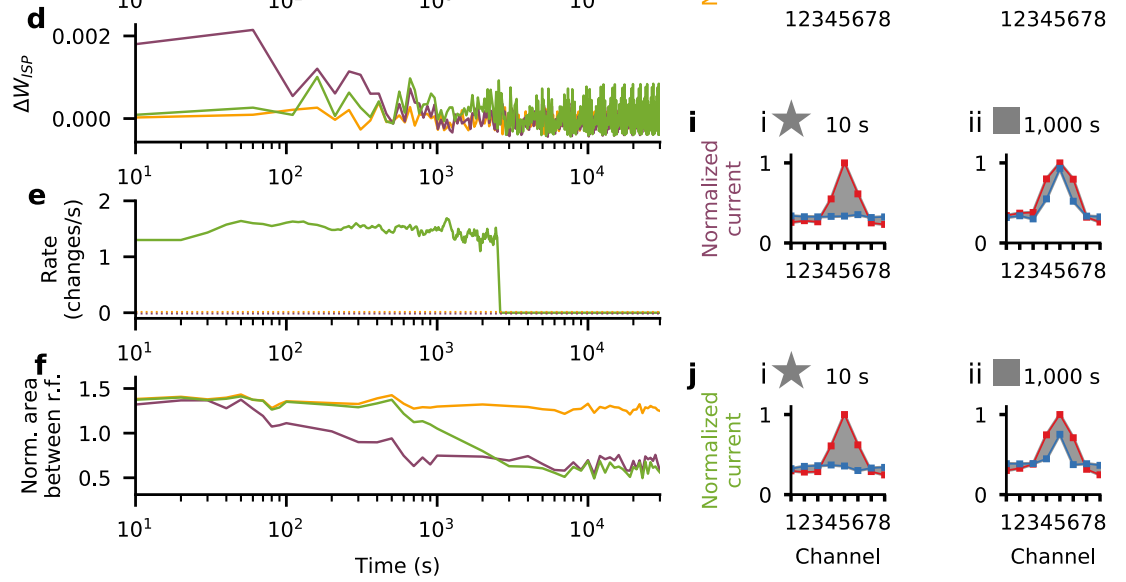

iii $10,000 \mathrm{~s}$
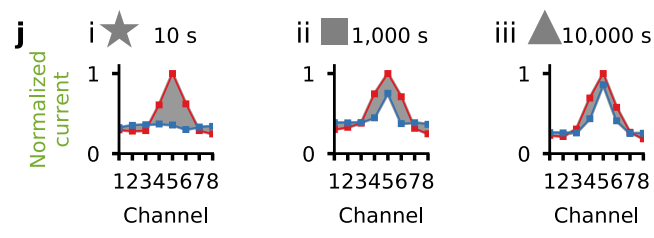

Figure S2. Developmental STP model with depression and facilitation normalized to the steady-state firing rate at $\mathbf{5 H z}$ input. (a) Schematic of our developmental short-term plasticity (STP) model (cf. Fig. S1); top: young and adult STP (as in Fig. 1); bottom: gradual changes in STP from depressing to facilitating dynamics (orange and purple respectively, in log-scale as in b-f). (b-f) Different variables of the model across simulated development for three different models: fixed short-term depression (fixed-STD, orange), fixed short-term facilitation (fixed-STF, purple) and developmental model with gradual changes in STP (dev-STP, green line). Note x-axis on log-scale. (b) Receiver neuron firing rate. (c) Mean inhibitory weight. (d) Mean changes in the weight of the inhibitory synaptic afferents. (e) Rate of STP change (note that both fixed-STF and STD remain fixed, shown as dashed lines). (f) Area between normalised excitatory and inhibitory tuning curves (cf. $h$-j) during the course of simulated development. A normalised area close to 0 represents a perfectly balanced neuron. (g) Additional statistics for the three models. (i) Total neuronal activity calculated using the area between the firing rate in (b) and the desired target rate of $5 \mathrm{~Hz}$. (ii) Average coefficient of variation of the firing rates across simulated development (cf. (b)). (iii) Percent of time spent under homeostasis (i.e. at the desired firing rate; cf. (b)). (iv) Average change in inhibitory weights (cf. (d)). (h-j) Snapshots of excitatory and inhibitory tuning curves across three points in simulated development: 10s (star), 1000s (square) and 10 000s (triangle). Shaded gray area represents difference between excitatory and inhibitory tuning curves (cf. (f)). (h-j) Excitatory (red) and inhibitory (blue) postsynaptic tuning curve for the fixed-STD (h), fixed-STF (i) and dev-STP models (j). 
bioRxiv preprint doi: https://doi.org/10.1101/2021.02.23.431593; this version posted March 12, 2021. The copyright holder for this preprint (which was not certified by peer review) is the author/funder, who has granted bioRxiv a license to display the preprint in perpetuity. It is made available under aCC-BY 4.0 International license.
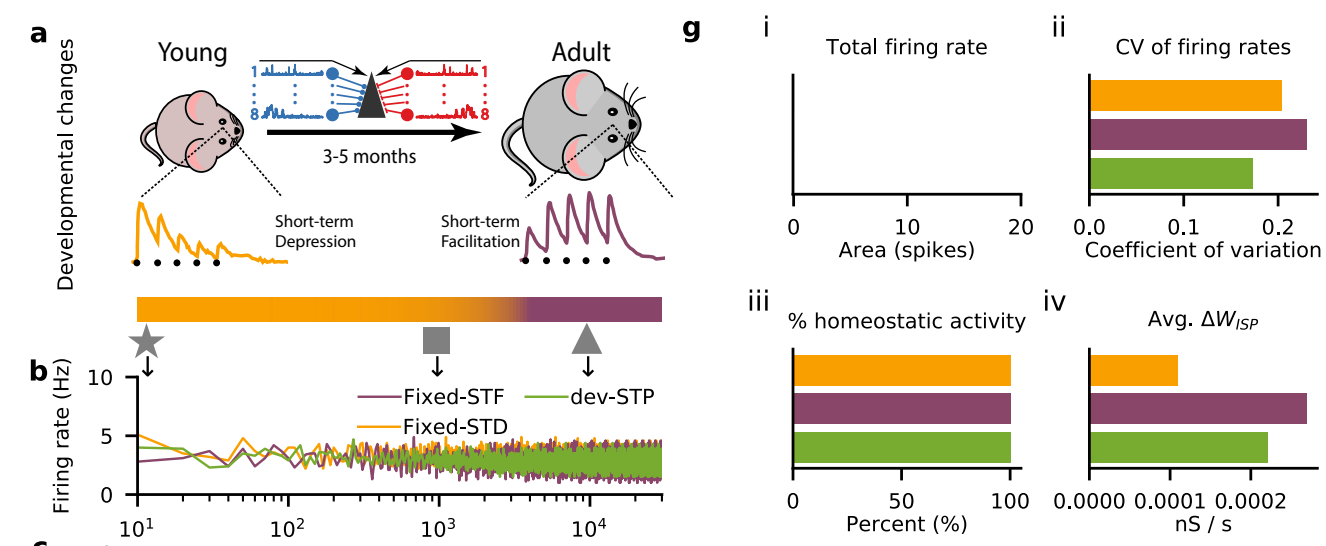

iii
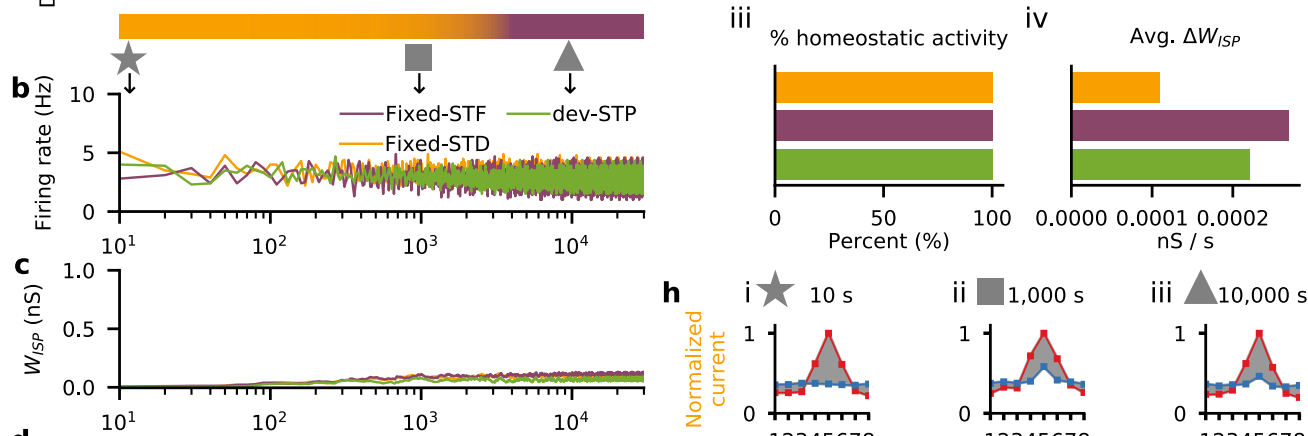

h

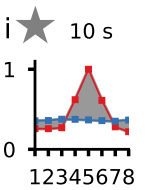

ii $\square 1,000 \mathrm{~s}$

iii $\triangle 10,000 \mathrm{~s}$
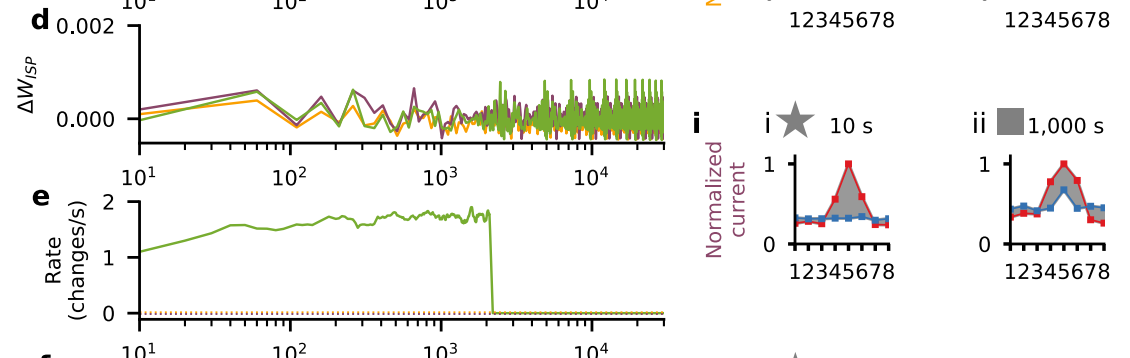

iii $10,000 \mathrm{~s}$
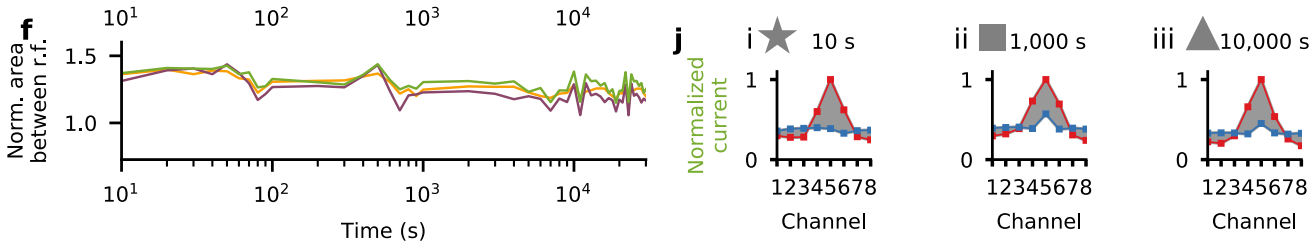

Figure S3. Developmental STP model with depression and facilitation normalized to the steady-state firing rate at $10 \mathrm{~Hz}$ input. (a) Schematic of our developmental short-term plasticity (STP) model (cf. Fig. S1); top: young and adult STP (as in Fig. 1); bottom: gradual changes in STP from depressing to facilitating dynamics (orange and purple respectively, in log-scale as in b-f). (b-f) Different variables of the model across simulated development for three different models: fixed short-term depression (fixed-STD, orange), fixed short-term facilitation (fixed-STF, purple) and developmental model with gradual changes in STP (dev-STP, green line). Note x-axis on log-scale. (b) Receiver neuron firing rate. (c) Mean inhibitory weight. (d) Mean changes in the weight of the inhibitory synaptic afferents. (e) Rate of STP change (note that both fixed-STF and STD remain fixed, shown as dashed lines). (f) Area between normalised excitatory and inhibitory tuning curves (cf. h-j) during the course of simulated development. A normalised area close to 0 represents a perfectly balanced neuron. (g) Additional statistics for the three models. (i) Total neuronal activity calculated using the area between the firing rate in (b) and the desired target rate of $5 \mathrm{~Hz}$. (ii) Average coefficient of variation of the firing rates across simulated development (cf. (b)). (iii) Percent of time spent under homeostasis (i.e. at the desired firing rate; cf. (b)). (iv) Average change in inhibitory weights (cf. (d)). (h-j) Snapshots of excitatory and inhibitory tuning curves across three points in simulated development: 10s (star), 1000s (square) and 10 000s (triangle). Shaded gray area represents difference between excitatory and inhibitory tuning curves (cf. (f)). (h-j) Excitatory (red) and inhibitory (blue) postsynaptic tuning curve for the fixed-STD (h), fixed-STF (i) and dev-STP models (j). 
bioRxiv preprint doi: https://doi.org/10.1101/2021.02.23.431593; this version posted March 12, 2021. The copyright holder for this preprint (which was not certified by peer review) is the author/funder, who has granted bioRxiv a license to display the preprint in perpetuity. It is made available under aCC-BY 4.0 International license.
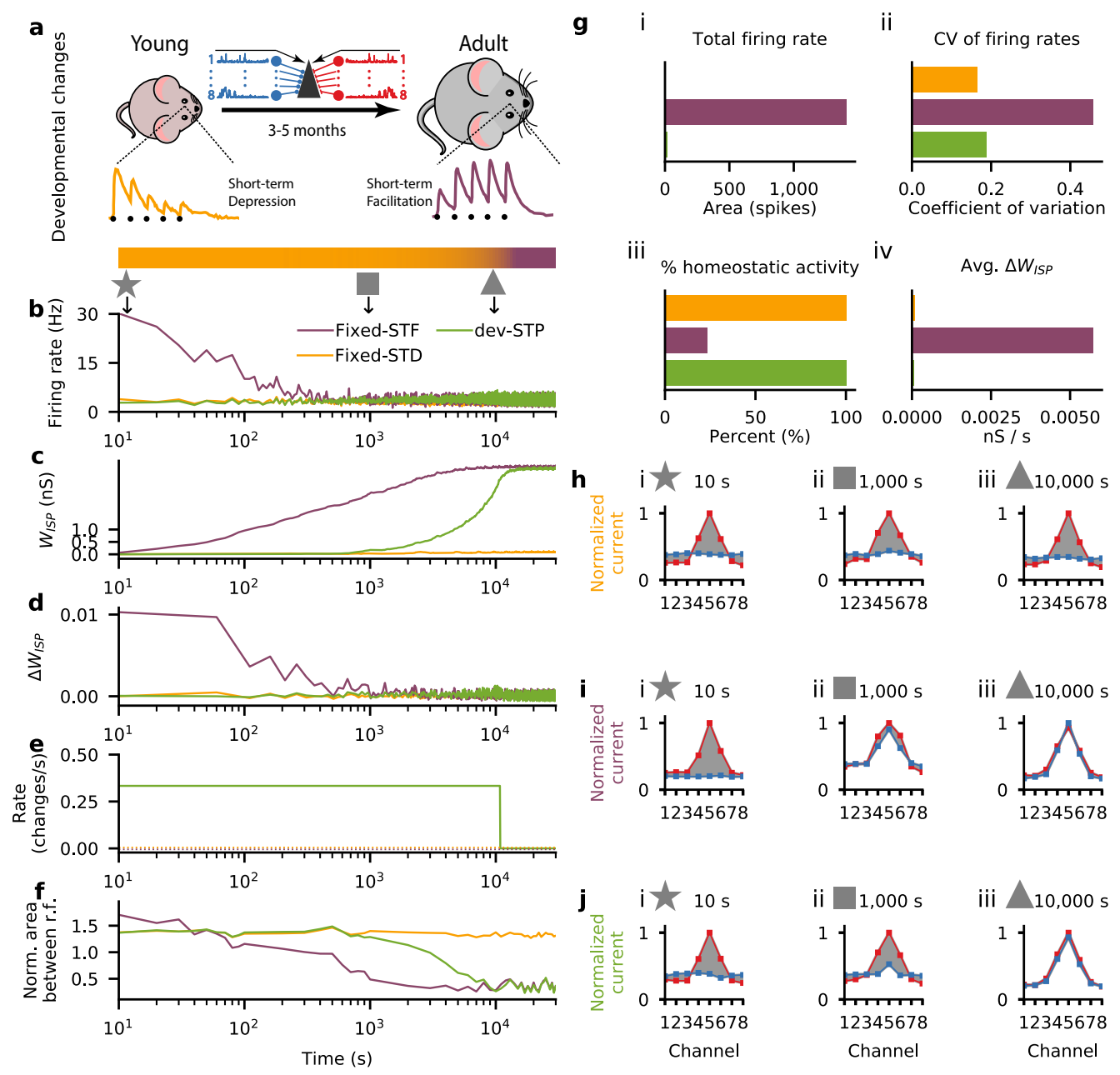

iii $10,000 \mathrm{~s}$
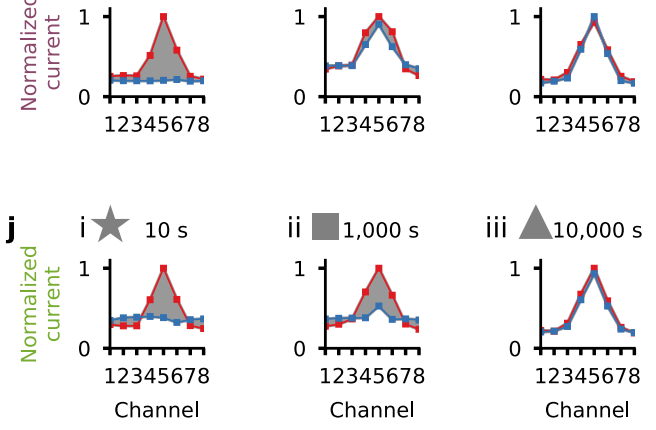

Figure S4. Developmental STP model in which STP changes are pre-defined. (a) Schematic of our developmental short-term plasticity (STP) model (cf. Fig. S1); top: young and adult STP (as in Fig. 1); bottom: gradual changes in STP from depressing to facilitating dynamics (orange and purple respectively, in log-scale as in b-f). (b-f) Different variables of the model across simulated development for three different models: fixed short-term depression (fixed-STD, orange), fixed short-term facilitation (fixed-STF, purple) and developmental model with gradual changes in STP (dev-STP, green line). Note x-axis on log-scale. (b) Receiver neuron firing rate. (c) Mean inhibitory weight. (d) Mean changes in the weight of the inhibitory synaptic afferents. (e) Rate of STP change (note that both fixed-STF and STD remain fixed, shown as dashed lines). (f) Area between normalised excitatory and inhibitory tuning curves (cf. h-j) during the course of simulated development. A normalised area close to 0 represents a perfectly balanced neuron. (g) Additional statistics for the three models. (i) Total neuronal activity calculated using the area between the firing rate in (b) and the desired target rate of $5 \mathrm{~Hz}$. (ii) Average coefficient of variation of the firing rates across simulated development (cf. (b)). (iii) Percent of time spent under homeostasis (i.e. at the desired firing rate; cf. (b)). (iv) Average change in inhibitory weights (cf. (d)). (h-j) Snapshots of excitatory and inhibitory tuning curves across three points in simulated development: 10s (star), 1000s (square) and 10 000s (triangle). Shaded gray area represents difference between excitatory and inhibitory tuning curves (cf. (f)). (h-j) Excitatory (red) and inhibitory (blue) postsynaptic tuning curve for the fixed-STD (h), fixed-STF (i) and dev-STP models (j). 
bioRxiv preprint doi: https://doi.org/10.1101/2021.02.23.431593; this version posted March 12, 2021. The copyright holder for this preprint (which was not certified by peer review) is the author/funder, who has granted bioRxiv a license to display the preprint in perpetuity. It is made available under aCC-BY 4.0 International license.
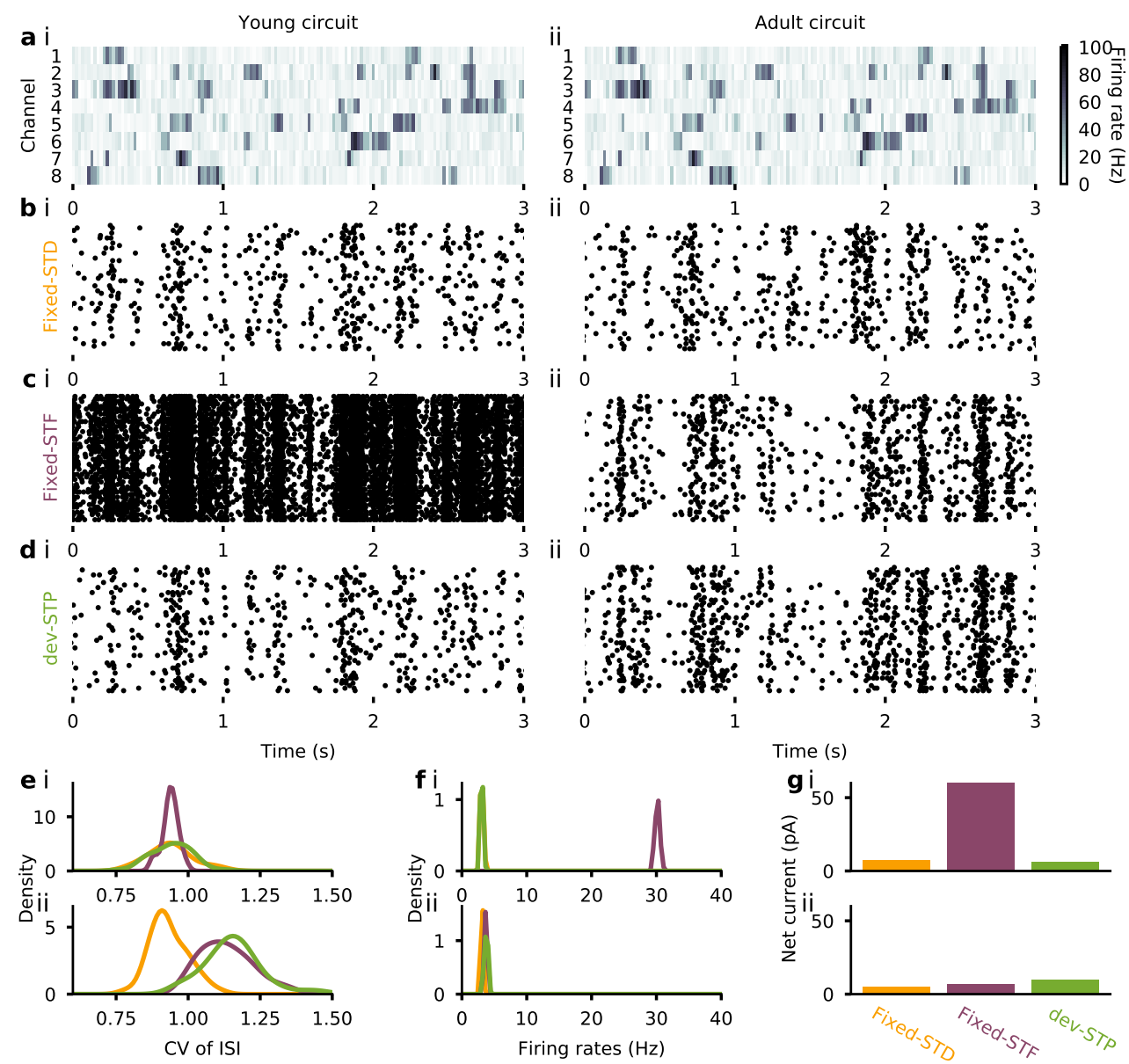

3 ii 0
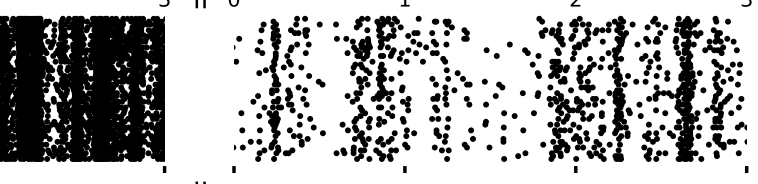

3 ii
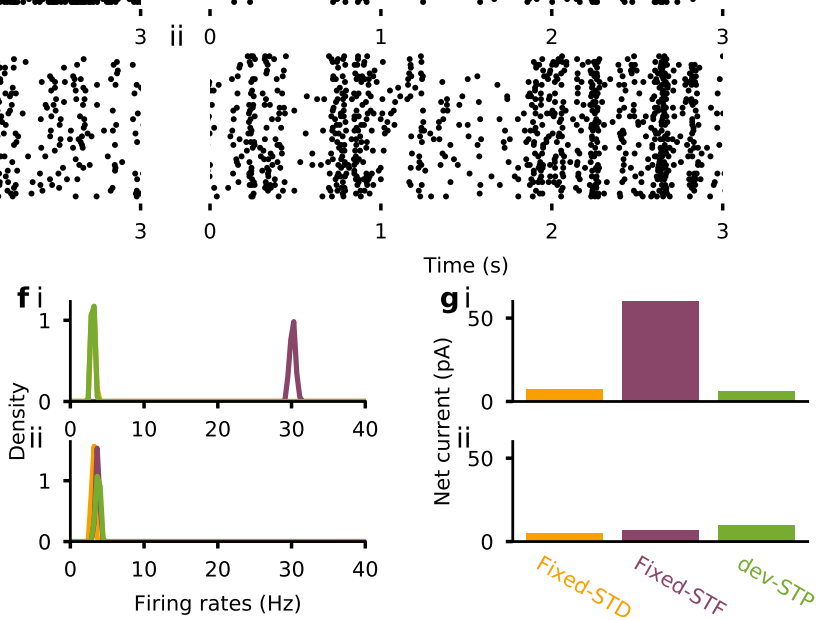

Figure S5. Developmental STP shapes firing statistics. (a) Input activity for each of the 8 channels over 3 seconds. Activity at the start of simulated development ( $i$, young condition) and after 8 hours of simulation (ii, adult condition) as in Fig. 2; color code represents firing rate of input. (b-d) Raster plot of receiver neuron for fixed-STD model (b), fixed-STF (c) and developmental STP model (d). (e-g) Summary statistics of the three models (as in b-d) for both young (i) and adult conditions (ii). (e) Coefficient of variation of the inter-spike intervals. (f) Average firing rates of the receiver neuron over 50 trials. (g) Average net current of the receiver neuron. 

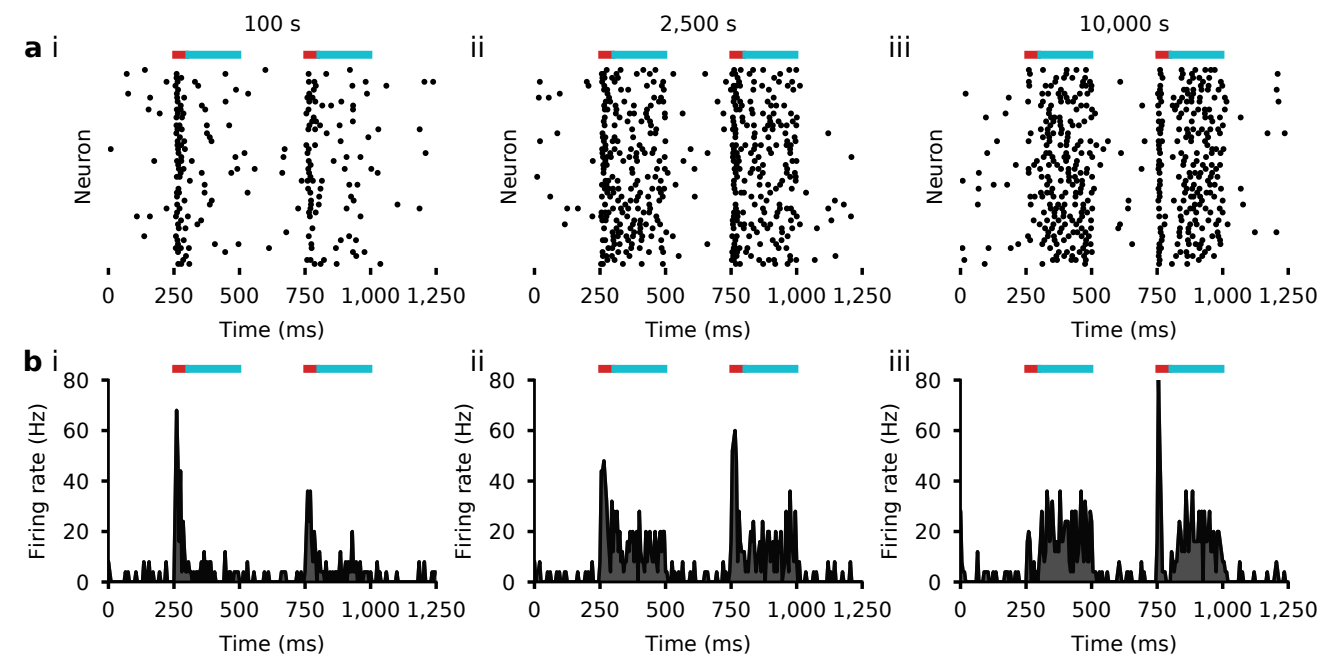

Figure S6. Spike rasters for step inputs at various snapshots. (a) Spike responses to two $150 \mathrm{~Hz}$ step inputs to the preferred channel when using the dev-STP model at 100s (i), 2,500s (ii), and 10,000s (iii); color bars on top represent the time at which the step inputs were given; the first $50 \mathrm{~ms}$ corresponds to the phasic activity (red), and the rest of the input time period to the tonic activity (cyan); b Firing rates of the spikes in (a) averaged across trials using $5 \mathrm{~ms}$ bins. 